\title{
An Initial Examination of Using Pseudospectral Methods for Timescale and Differential Geometric Analysis of Nonlinear Optimal Control Problems
}

\author{
Christopher L. Darby* \\ Anil V. Rao ${ }^{\dagger}$ \\ Department of Mechanical and Aerospace Engineering \\ University of Florida \\ Gainesville, FL 32611
}

\begin{abstract}
An initial examination of the development of a framework for analyzing the time-scale and differential geometric structure of nonlinear optimal control problems is considered. The framework is synthesized by combining a recently developed direct collocation method called the Gauss pseudospectral method (GPM) with concepts from differential geometry. In particular, the GPM is known to provide optimal state and costate information, thus enabling the computation of accurate Hamiltonian phase space trajectories. Using the optimal Hamiltonian phase space trajectories from the GPM, it is possible to analyze the timescale and differential geometric structure by computing the finite-time Lyapunov exponents and Lyapunov vectors. The Lyapunov exponents provide information about both the stable/unstable and slow/fast behavior of the Hamiltonian system along the optimal trajectory. Furthermore, the directions in the phase space along which these different behaviors act are isolated by decomposing the tangent space of the Hamiltonian system using the finitetime Lyapunov vectors. The approach is demonstrated successfully on two examples. The main contribution of this paper is to demonstrate the effectiveness of combining the Gauss pseudospectral method with results from differential geometry to assess the structure of optimally controlled systems, but without having to solve the Hamiltonian boundary-value problem that arises from the calculus of variations.
\end{abstract}

\section{Introduction}

Due to the growing complexity of engineering applications, analytic methods for studying nonlinear dynamical systems are becoming increasingly difficult. In particular, optimal control is a subject where such analytical tractability has diminished due to the extraordinary complexity of new applications. Unfortunately, as the complexity of applications grows, the need to understand the structure of optimally controlled systems becomes even more important. For example, where it may have previously been difficult or impossible, advanced computational methodologies can potentially provide insight into the structure of the solution to an optimal control problem

${ }^{*}$ Graduate Student, Department of Mechanical and Aerospace Engineering. E-mail: cdarby@ufl.edu

${ }^{\dagger}$ Assistant Professor, Department of Mechanical and Aerospace Engineering. E-mail: anilvrao@ufl.edu. Corresponding Author. 
and lead to simplifications which in turn can lead to the development of simplified near-optimal guidance laws for complex dynamical systems.

Historically, numerical methods for solving optimal control problems have fallen into two categories: indirect methods and direct methods. ${ }^{1}$ In an indirect method, the first order necessary conditions for optimality from the calculus of variations are derived and the resulting Hamiltonian boundary-value problem (HBVP) must be solved. The advantage of an indirect methods is that, if a solution can be found, the local optimality of the solution can be verified. In addition, because a solution from an indirect method provides accurate costate information, it is possible to extract a great deal of insight from the solution obtained via an indirect method. Indirect methods, however, have small radii of convergence and become increasingly difficult to employ as the complexity of the problem increases. Finally, even in the case where the optimality conditions can be derived, solving the resulting HBVP may be difficult if not impossible. Well-known indirect methods include shooting methods ${ }^{2}$ multiple shooting methods, ${ }^{3,4}$ and neighboring extremal methods. ${ }^{5}$

In a direct method the continuous-time optimal control problem is transcribed into a nonlinear program (NLP). This nonlinear program is then solved numerically by one of a number of well known software tools (e.g., SNOPT, ${ }^{6} \mathrm{KNITRO}^{7}$ or SPRNLP ${ }^{8}$ ). The objective of the NLP is to satisfy a set of conditions called the Karush-Kuhn-Tucker (KKT) conditions. ${ }^{9}$ While direct methods typically have larger radii of convergence as compared to indirect methods and do not require derivation of the first-order optimality conditions, in general the KKT multipliers of the NLP do not map accurately to the costates associated with the Hamiltonian system. As a result, it is difficult to verify that the solution obtained from a direct method is truly an extremal solution. Furthermore, additional post-optimality analysis becomes increasingly unreliable when using the results obtained from a direct method.

In recent years the class of pseudospectral or orthogonal collocation methods have risen to prominence in computational optimal control. A key feature of pseudospectral methods is that they provide all of the benefits of direct collocation methods together with the ability to obtain highaccuracy estimates of the costate (adjoint). Three of the more commonly used pseudospectral methods are the Legendre pseudospectral method (LPM), ${ }^{10-12}$ the Radau pseudospectral method (RPM), ${ }^{12,13}$ and the Gauss pseudospectral method (GPM). ${ }^{12,14-19}$ A comparison between the three aforementioned pseudospectral methods is given in Ref. 18. The key difference between the three methods is that in the LPM the dynamics are collocated at both endpoints, in the RPM the dynamics are collocated at only one endpoint, and in the GPM the dynamics are collocated at neither endpoint. Finally, of the three methods, the GPM is the only one that provides an equivalence between the costates of the optimal control problem and the KKT multipliers of the NLP. While any of the three methods can be used, in this research we utilize the GPM because of its demonstrated ability to compute highly accurate costates in an algebraically simple manner using the KKT conditions of the NLP. $14,15,17$

A common feature of many optimal control problems is that they either evolve on multiple time-scales or have solution segments that lie in lower-dimensional spaces from the space in which the problem is posed. As a result, it is often possible to determine simplified approximations to solutions of optimal control problems where the simplified solution has nearly the same performance and exhibits the key characteristics of the exact solution. Historically, multiple time-scale dynamical systems have been analyzed using singular perturbation methods. ${ }^{20,21}$ Typically, the dynamics are cast in the standard singularly perturbed form where the slow and fast variables can be explicitly identified via determination of a small parameter that determines the time-scale separation. $^{20,21}$ Using singular perturbation methods it is possible to determine reduced-order approximations to the solution. In special cases where the dynamics may not be originally cast in 
the standard form it may be possible to transform the dynamics into the standard form. ${ }^{20,21}$ If the standard form is attainable and the problem has a boundary-layer-type structure, techniques such as matched asymptotic expansions can then be used to determine approximate inner and outer solutions on the various time-scales of interest.

While singular perturbation methodology is an extremely useful and proven analysis tool, it is limited to problems that can ultimately be cast in the standard form. Unfortunately, general nonlinear dynamical systems cannot be cast in the standard form. This last fact, together with the growing complexity of current and future applications, makes it necessary to develop approaches for multiple time-scale problems for which singular perturbation theory is not wellsuited. One numerical approach that has been developed for time-scale identification of problems with a boundary-layer-type structure is the computational singular perturbation (CSP) methodology. ${ }^{22-24}$ In CSP, the dynamics are integrated explicitly and time-scales are eliminated as they become dormant. In particular, when the activity of particular time-scales decreases below a specified threshold, these time-scales are eliminated by projecting the solution into a lower-dimensional manifold that retains only the active time-scale. A key assumption about CSP is that the dynamics are asymptotically stable. As a result, the methodology is most applicable to problems where the dynamics eventually reach equilibrium (e.g., chemical kinetics problems). While CSP has been successful for problems with asymptotically stable dynamics, Hamiltonian systems have both stable and unstable dynamics. It is noted that extensions to CSP for Hamiltonian systems have been developed, but these extensions have focused on the special case of hyper-sensitive optimal control problems ${ }^{25-27}$ (where the time interval of interest is significantly longer than the time-scales in the problem). The CSP methodology has not, however, been extended to the general optimal control problem with path constraints and interior point constraints.

In recent years, a general theory for analyzing the time-scale and differential geometric structure of nonlinear dynamical systems has been developed. ${ }^{28}$ In particular, Ref. 28 develops the fact that the finite-time Lyapunov exponents ${ }^{29}$ provide approximations to the time-scale and stability of a nonlinear dynamical system at a general point in the state space. Correspondingly, the finite-time Lyapunov vectors provide the directions in the state space along which the time-scales and stability act. The methodology proposed in Ref. 28 utilizes a result from the work of Lorenz ${ }^{30}$ where it was found that the finite-time Lyapunov exponents and their corresponding directions (i.e., the finite-time Lyapunov vectors) can be determined by a singular value decomposition of the transition matrix of the linearized dynamics along a trajectory of the nonlinear system. Mease ${ }^{28}$ has shown that a singular value decomposition of the transition matrix of the associated linearized dynamics of a nonlinear dynamical system over a finite time will provide an approximation to the true (asymptotic) Lyapunov exponents and vectors if the averaging time is chosen properly (refer to $^{28,31}$ for more details on the asymptotic Lyapunov exponents and vectors). In the case of an optimal control problem, the time-scales and differential geometry can be obtained by analyzing the the combined state/costate dynamics (i.e., analyzing the linearized dynamics of the Hamiltonian system that arises from the calculus of variations).

In this paper, an approach is conceived to analyze the time-scales and differential geometric structure of general constrained nonlinear optimal control problems. The framework is synthesized by combining pseudospectral methods for solving optimal control problems with results from nonlinear dynamical system theory. In particular, in this paper the time-scales and differential geometric structure of a nonlinear Hamiltonian system are analyzed using the approach described in Ref. 28 and 31. In order to analyze the structure of the Hamiltonian phase space, extremal trajectories of a nonlinear optimal control problem are computed using the Gauss pseudospectral method. ${ }^{14,15,17}$ The tangent space to the Hamiltonian phase space can then be decomposed using an orthonormal basis of finite-time Lyapunov vectors. By analyzing the contribution 
of the Hamiltonian vector field along the various Lyapunov vectors, it is possible to determine if simplification to the optimal solution is feasible. The approach developed in this paper is applied to two examples to demonstrate its utility. It is found that the approach is viable and is the starting point for model decomposition of nonlinear optimal control problems that evolve either on multiple time-scales or in reduced manifolds of the Hamiltonian phase space.

\section{Optimal Control Problem in Bolza Form}

Without loss of generality, consider the following optimal control problem in Bolza form. Minimize the cost functional

$$
J=\boldsymbol{\Phi}\left(x(-1), t_{0}, \mathbf{x}(-1), t_{f}\right)+\frac{t_{f}-t_{0}}{2} \int_{-1}^{1} \mathcal{L}(\mathbf{x}(\tau), \mathbf{u}(\tau), \tau) d \tau
$$

subject to the dynamic constraints

$$
\frac{d \mathbf{x}}{d \tau}=\frac{t_{f}-t_{0}}{2} f(\mathbf{x}(\tau), \mathbf{u}(\tau), \tau)
$$

the boundary conditions (i.e., the event constraints)

$$
\phi\left(\mathbf{x}(-1), t_{0}, \mathbf{x}(1), t_{f}\right)=\mathbf{0}
$$

and the inequality path constraints

$$
\mathbf{C}\left(\mathbf{x}(\tau), \mathbf{u}(\tau), \tau ; t_{0}, t_{f}\right) \leq \mathbf{0}
$$

where $\mathbf{x}$ is the state, $\mathbf{u}$ are the controls and $\tau$ is time. The first order optimality conditions for the optimal control problem given in Eqs. (1)-(4) are given as

$$
\begin{gathered}
\frac{d \mathbf{x}}{d \tau}=\frac{t_{f}-t_{0}}{2} \frac{\partial H}{\partial \boldsymbol{\lambda}} \\
\frac{d \boldsymbol{\lambda}}{d \tau}=-\frac{t_{f}-t_{0}}{2} \frac{\partial H}{\partial \mathbf{x}} \\
\frac{\partial H}{\partial \mathbf{u}}=\mathbf{0} \\
\phi\left(\mathbf{x}(-1), t_{0}, \mathbf{x}(1), t_{f}\right)=\mathbf{0} \\
\boldsymbol{\lambda}(-1)=-\frac{\partial \boldsymbol{\Phi}}{\partial \mathbf{x}\left(\tau_{0}\right)}+\boldsymbol{\nu}^{T} \frac{\partial \phi}{\partial \mathbf{x}\left(\tau_{0}\right)}, \quad \boldsymbol{\lambda}(1)=\frac{\partial \boldsymbol{\Phi}}{\partial \mathbf{x}\left(\tau_{f}\right)}-\boldsymbol{\nu}^{T} \frac{\partial \phi}{\partial \mathbf{x}\left(\tau_{f}\right)} \\
H(-1)=\frac{\partial \Phi}{\partial t_{0}}-\boldsymbol{\nu}^{T} \frac{\partial \phi}{\partial t_{0}}, \quad H(1)=-\frac{\partial \Phi}{\partial t_{f}}+\boldsymbol{\nu}^{T} \frac{\partial \phi}{\partial t_{f}} \\
\mu_{j}(\tau)=0, \quad \mu_{j}(\tau) \leq 0, \quad j=1, \ldots, c
\end{gathered}
$$

where $H$ is the augmented Hamiltonian given as

$$
H=\mathcal{L}+\boldsymbol{\lambda}^{T} f-\boldsymbol{\mu}^{T} \mathbf{C}
$$

It should be noted that the time interval $\tau \in[-1,1]$ can be transformed to the time interval $t \in$ $\left[t_{0}, t_{f}\right]$ by the affine transformation:

$$
t=\frac{t_{f}-t_{0}}{2} \tau+\frac{t_{f}+t_{0}}{2}
$$




\section{Gauss Pseudospectral Method}

In this research the Gauss pseudospectral method $(\mathrm{GPM})^{15}$ is used to compute primal (i.e., state and control) and dual (i.e., costate) solutions to nonlinear optimal control problems. While the GPM has been described elsewhere, ${ }^{15,19}$ a brief discussion is provided here for completeness of the discussion. First, the state, $\mathbf{x}(\tau)$ is approximated by a basis of $N+1$ Lagrange interpolating polynomials, $L, 32$ as

$$
\mathbf{x}(\tau) \approx \mathbf{X}(\tau)=\sum_{i=0}^{N} \mathbf{X}\left(\tau_{i}\right) L_{i}(\tau)
$$

where

$$
L_{i}(\tau)=\prod_{j=0, j \neq i}^{N} \frac{\tau-\tau_{j}}{\tau_{i}-\tau_{j}}
$$

Differentiating Eq. (14), we obtain

$$
\dot{\mathbf{x}}(\tau) \approx \dot{\mathbf{X}}(\tau)=\sum_{i=0}^{N} \mathbf{x}\left(\tau_{i}\right) \dot{L}_{i}(\tau)
$$

The derivative of the Lagrange polynomial at the $N$ Legendre-Gauss points [i.e., the roots of the $N^{\text {th }}$ degree Legendre polynomial, $\left.P_{N}(\tau)\right]$ is represented by a differentiation matrix, $D \in \mathbb{R}^{N \times N+1}$ as $^{16}$

$$
D_{k i}=\dot{L}_{i}\left(\tau_{k}\right)=\left\{\begin{array}{cc}
\frac{\left(1+\tau_{k}\right) \dot{P}_{N}\left(\tau_{k}\right)+P_{N}\left(\tau_{k}\right)}{\left(\tau_{k}-\tau_{i}\right)\left[\left(1+\tau_{i}\right) \dot{P}_{N}\left(\tau_{i}\right)+P_{N}\left(\tau_{i}\right)\right]}, & \\
\frac{\left(1+\tau_{i}\right) \ddot{P}_{N}\left(\tau_{i}\right)+2 \dot{P}_{N}\left(\tau_{i}\right)}{2\left[\left(1+\tau_{i}\right) \dot{P}_{K}\left(\tau_{i}\right)+P_{N}\left(\tau_{i}\right)\right]} & , \quad i=k
\end{array} \quad \begin{array}{ll}
(i=0, \ldots, N) \\
\end{array}\right.
$$

The dynamics of the continuous time optimal control problem are then transcribed at the LG points into the following set of algebraic constraints:

$$
\sum_{i=0}^{N} D_{k i} X_{i}-\frac{t_{f}-t_{0}}{2} \mathbf{f}\left(\mathbf{x}_{k}, \mathbf{U}_{k}, \tau_{k}, t_{o}, t_{f}\right)=\mathbf{0} \quad k=(1, \ldots, N)
$$

where $\mathbf{X}_{k} \equiv \mathbf{X}\left(\tau_{k}\right) \in \mathbb{R}^{n}$ and $\mathbf{U}_{k} \equiv \mathbf{U}\left(\tau_{k}\right) \in \mathbb{R}^{m},(k=1, \ldots, N)$. Next, the following integral constraint, approximated in the form of a Gaussian quadrature, is used to relate the initial state $\mathbf{X}_{0}=\mathbf{X}(-1)$ to $\mathbf{X}_{f}=\mathbf{X}(1)$ :

$$
\mathbf{X}_{f} \equiv \mathbf{X}_{0}+\frac{t_{f}-t_{0}}{2} \sum_{k=1}^{N} \omega_{k} \mathbf{f}\left(\mathbf{X}_{k}, \mathbf{U}_{k}, \tau_{k}, t_{0}, t_{f}\right)
$$

where $w_{k}$ are the Gauss weights. Furthermore, the cost functional is approximated by a cost function as

$$
J=\boldsymbol{\Phi}\left(\mathbf{X}_{0}, t_{0}, \mathbf{X}_{f}, t_{f}\right)+\frac{t_{f}-t_{0}}{2} \sum_{k=1}^{N} w_{k} \mathbf{f}\left(\mathbf{X}_{k}, \mathbf{U}_{k}, \tau_{k}, t_{0}, t_{f}\right)
$$

Next, the boundary conditions are given as:

$$
\phi\left(\mathbf{X}_{0}, t_{0}, \mathbf{X}_{f}, t_{f}\right)=\mathbf{0}
$$


Finally, the path constraint is discretized at the LG points as

$$
\mathbf{C}\left(\mathbf{X}_{k}, \mathbf{U}_{k}, \tau_{k}, t_{o}, t_{f}\right) \leq \mathbf{0} \quad(k=1, \ldots, N)
$$

Eqs. (18)-(22) are the NLP whose solution is the approximation to the continuous time optimal control problem.

Next, the first order optimality conditions of the NLP, or the KKT conditions, are obtainable from the augmented cost functional. The augmented cost functional is formed from the Lagrange multipliers $\tilde{\boldsymbol{\Lambda}}_{k} \in \mathbb{R}^{n}, \tilde{\boldsymbol{\mu}}_{k} \in \mathbb{R}^{c}, k=1, \ldots, N, \tilde{\boldsymbol{\Lambda}}_{F} \in \mathbb{R}^{n}$, and $\tilde{\boldsymbol{\nu}} \in \mathbb{R}^{q}$. The KKT conditions are found by setting the derivatives of the Lagrangian with respect to $\mathbf{X}_{0}, \mathbf{X}_{k}, \mathbf{X}_{f}, \mathbf{U}_{k}, \tilde{\boldsymbol{\Lambda}}_{k}, \tilde{\boldsymbol{\nu}}_{k}, \tilde{\Lambda}_{F}, \tilde{\boldsymbol{\nu}}, t_{0}$ and $t_{f}$ equal to zero. A more complete description of this process is given in Ref. 15.

In addition to being able to obtain the primal solution of the optimal control problem by solving the NLP of Eqs. (18)-(22), the dual (i.e., costate) solution of the NLP can be obtained via the following costate mapping: ${ }^{15}$

$$
\boldsymbol{\Lambda}_{k}=\frac{\tilde{\boldsymbol{\Lambda}}_{k}}{\omega_{k}}+\tilde{\boldsymbol{\Lambda}}_{F}, \quad \boldsymbol{\mu}_{k}=\frac{2}{t_{f}-t_{0}} \frac{\tilde{\boldsymbol{\nu}}_{k}}{\omega_{k}}, \quad \boldsymbol{\nu}=\tilde{\boldsymbol{\nu}}, \quad \boldsymbol{\Lambda}\left(t_{0}\right)=\tilde{\boldsymbol{\Lambda}}_{0}, \quad \boldsymbol{\Lambda}\left(t_{f}\right)=\tilde{\boldsymbol{\Lambda}}_{F}
$$

where $\boldsymbol{\Lambda}_{k},(k=1, \ldots, N)$ are the costate estimates at the LG points, $\boldsymbol{\Lambda}_{F}$ is the costate estimate at the terminal point, $\boldsymbol{\Lambda}_{0}$ is the initial costate estimate, $\tilde{\Lambda}_{k},(k=1, \ldots, N)$ are the KKT multipliers of the NLP of Eq. (18), $\tilde{\Lambda}_{F}$ is the KKT multiplier associated with the quadrature constraint of Eq. (19), $\boldsymbol{\nu}$ is the Lagrange multiplier associated with the boundary conditions, and $\boldsymbol{\mu}_{k},(k=1, \ldots, N)$ are the KKT multipliers associated with the constraints of Eq. (22). A more detailed description of the costate mapping can be found in Refs. 14-16.

\section{Nonlinear Time-Scales and Differential Geometry}

\section{A. Singular Perturbation Theory}

Many optimal control problems evolve on multiple time-scales or have the property that different segments of the solution lie in manifolds of lower dimension from the original $2 n$-dimensional Hamiltonian phase space. As a result, determination of the time-scale and differential geometric structure can lead to possible simplifications that in turn can be used to extract simplified guidance laws. Historically, analysis of multiple time-scale optimal control problems has required that the dynamics be given in the so-called standard singularly perturbed form given as ${ }^{20,21}$

$$
\begin{aligned}
& \dot{\mathbf{x}}=\mathbf{f}(\mathbf{x}, \mathbf{z}, \epsilon, t) \\
& \epsilon \dot{\mathbf{z}}=\mathbf{g}(\mathbf{x}, \mathbf{z}, \epsilon, t)
\end{aligned}
$$

where $\epsilon$ is a small parameter that occurs either naturally due to the dynamics of the system or is introduced artificially to determine if a time-scale separation exists. In the standard form, the variables $\mathbf{x}$ and $\mathbf{z}$ identify the slow and fast variables, respectively, in the system. For problems cast in the standard form and with a boundary-layer type structure, techniques such as the method of matched asymptotic expansions ${ }^{20,21}$ are then used to determine approximations to various segments of the solution. While the standard singularly perturbed form is useful for some problems, many nonlinear dynamical systems that evolve on multiple time-scales can neither be written naturally in the standard form nor can be transformed to the standard form. In such cases, singular perturbation theory is difficult to utilize and other techniques must be employed. For problems that cannot be cast in the standard form, more general techniques have been recently developed.

6 of 26

American Institute of Aeronautics and Astronautics 
In the remainder of this section we draw heavily from the work of Ref. 28 and 31 and show how one can analyze the time-scale and differential geometric structure of a Hamiltonian system that arises in optimal control.

\section{B. Time-Scales and Differential Geometry of Hamiltonian Systems}

Consider now an autonomous nonlinear dynamical system of the form

$$
\dot{\mathbf{p}}=\mathbf{G}(\mathbf{p})
$$

where $\mathbf{p}(t)=(\mathbf{x}(t), \boldsymbol{\lambda}(t)) \in \mathbb{R}^{2 n}$ is the combined state-costate (phase) vector for a Hamiltonian system of the form

$$
\begin{aligned}
& \dot{\mathbf{x}}=\partial H / \partial \boldsymbol{\lambda} \equiv H_{\boldsymbol{\lambda}} \\
& \dot{\boldsymbol{\lambda}}=-\partial H / \partial \mathbf{x} \equiv-H_{\mathbf{x}}
\end{aligned}
$$

Assume further that any trajectory $\mathbf{p}(t)$ of Eq. (25) evolves in a set $\chi \subset \mathbb{R}^{2 n}$, 31a. Next, let $\bar{t}$ be an averaging time (i.e., a time period over which the analysis of the time-scales and differential geometry will be assessed at a given point in the phase space ${ }^{28}$ ). Assume that a Euclidean metric is used throughout this paper. ${ }^{\mathrm{b}}$ Now, let $\mathbf{p}(t)=\phi(t, \mathbf{p})$ where $\phi$ satisfies the two conditions: (1) $\partial \phi(t, \mathbf{p}) / \partial t=\mathbf{f}[\phi(t, \mathbf{p})]$ for every value of $\mathbf{t}$, and (2) $\phi(0, \mathbf{p})=\mathbf{p}^{\mathrm{c}}{ }^{\mathrm{c}}$ The associated linear dynamical system for the original state space is given $a^{28}$

$$
\frac{\partial \mathbf{v}}{\partial t}=\mathbf{F}[\phi(t, \mathbf{p})] \mathbf{v}
$$

where $\mathbf{F}[\boldsymbol{\phi}(t, \mathbf{p})]=\partial \mathbf{G} / \partial \mathbf{p}$, and the rate coordinate vector $\mathbf{v}(t, \mathbf{p})$ is propagated $\mathrm{as}^{28}$

$$
\mathbf{v}(t, \mathbf{p})=\mathbf{\Phi}(t, \mathbf{p}) \mathbf{v}(0, \mathbf{p})
$$

where $\boldsymbol{\Phi}$ is the transition matrix of $\mathbf{F}$. The transition matrix also satisfies the fundamental equation

$$
\frac{\partial \boldsymbol{\Phi}}{\partial t}=\mathbf{F}[\phi(t, \mathbf{p})] \boldsymbol{\Phi}, \quad \boldsymbol{\Phi}(s, \mathbf{p})=I
$$

where $s$ is a time from which the propagation is performed along the trajectory $\mathbf{p}(t)$ in the phase space. $\boldsymbol{\Phi}$ is related to $\phi$ by $\boldsymbol{\Phi}(t, \mathbf{p})=\partial \phi(t, \mathbf{p}) / \partial \mathbf{p}$. Because $\mathbf{v}$ is a tangent vector, Eqs. (25) and (27) describe how a point $(\mathbf{p}, \mathbf{v})$ evolves in a $4 n$-dimensional tangent bundle $T \chi$. For each $\mathbf{p} \in \chi$, there is an associated tangent space $T_{\mathbf{p}} \chi$, the space of all possible vectors tangent to orbits passing through p.

Using the transition matrix $\Phi$, approximations to the time-scales and differential geometric structure at a given point in the Hamiltonian phase space can be obtained by performing a singular-value decomposition ${ }^{\mathrm{d}}$ of $\boldsymbol{\Phi}(s+\bar{t}, \mathbf{p})$ as

$$
\mathbf{\Phi}=\mathbf{N} \Sigma \mathbf{L}^{T}
$$

Assuming that the entries of $\Sigma$ are distinct, the matrices $\mathbf{N}$ and $\mathbf{L}$ are uniquely determined. ${ }^{33 e}$ The singular values may be thought of as expansion or contraction factors of a unit hypersphere in the

\footnotetext{
${ }^{a}$ For the asymptotic Lyapunov theory, the set needs to be compact. For the finite time theory, this is not necessary.

${ }^{\mathrm{b}} \mathrm{A}$ Euclidean metric is constant along solutions, therefore, any exponential expansion or contraction of tangent vectors to the solution is due to the dynamics. ${ }^{28}$

${ }^{\mathrm{c}}$ The notation $\phi(t, \mathbf{p})$ is used instead of $\mathbf{p}(t)$ because $\mathbf{p}$ is being used for the initial condition

${ }^{\mathrm{d}}$ Without loss of generality, assume the singular values are ordered in descending order.

${ }^{\mathrm{e}}$ In this paper we assume for simplicity that the singular values are distinct such that the SVD defines a nondegenerate spectrum with uniformly bounded gaps.
} 
$2 n$-dimensional tangent space along principle orthogonal directions into a hyper-ellipse. With the assumption of distinct singular values, we obtain the following filtration of nested subspaces of $T_{\mathbf{p}} \chi^{.28}$

$$
0=\mathbf{L}_{0} \subset \mathbf{L}_{1} \subset \mathbf{L}_{2} \subset \ldots \subset \mathbf{L}_{2 n}=T_{\mathbf{p}} \chi
$$

The subspaces can be represented by the column vectors of $\mathbf{L}$ as

$$
\mathbf{L}_{1}=\operatorname{span}\left(\mathbf{l}_{1}\right), \mathbf{L}_{2}=\operatorname{span}\left(\mathbf{l}_{1}, \mathbf{l}_{2}\right), \ldots, \mathbf{L}_{2 n}=\operatorname{span}\left(\mathbf{l}_{1}, \ldots, \mathbf{l}_{2 n}\right)
$$

If we allow $\mathbf{v}$ to be propagated for $\bar{t}$ time units, then the finite time Lyapunov exponents can be determined from the singular values of $\boldsymbol{\Phi}(s+\bar{t}, \mathbf{p})$ as ${ }^{28,30}$

$$
\mu_{i}(s+\bar{t}, \mathbf{p})=\frac{1}{\bar{t}} \log \sigma_{i}(s+\bar{t}, \mathbf{p})
$$

where $\sigma_{i}$ are the singular values from $\Sigma$. These are the exponential rates associated with the $2 n$ dimensional principle directions along which the solution evolves starting from the time $s$ along the trajectory $\mathbf{p}(t)$. Furthermore, the column vectors of $\mathbf{L},\left(\mathbf{1}_{1}, \mathbf{1}_{2}, \ldots, \mathbf{1}_{2 n}\right)$ are the finite time Lyapunov vectors associated with the Lyapunov exponents. The finite-time Lyapunov exponents are approximations of the infinite time Lyapunov exponents, i.e., the exponents obtained by taking the limit as $\bar{t} \rightarrow \infty$ in Eq. (33), where the infinite time Lyapunov exponents define the true time-scales and stability at each point in the phase space. ${ }^{f}$ Correspondingly, the finite-time Lyapunov vectors are approximations to the infinite-time Lyapunov vectors where the infinite-time Lyapunov vectors define the directions along which the different time-scales act. Therefore, for a sufficiently large value of $\bar{t}$, the finite time Lyapunov exponents will provide a good approximation to the time-scales and stability of the system and the corresponding Lyapunov vectors will provide a good approximation to the directions along which these different behaviors act. In order to ensure that $\bar{t}$ is sufficiently large, the following rule is applied:

$$
\bar{t}>\frac{3}{\nabla \mu}
$$

where $\nabla \mu$ is the spectral gap separating either the slow and fast and/or stable and unstable behavior. Eq. (34) is employed because if the neighboring Lyapunov exponents $\mu_{j}(s+\bar{t}, \mathbf{p})$ and $\mu_{j+1}(s+$ $\bar{t}, \mathbf{p})$ are distinct, then as $\bar{t}$ increases, the subspace $L_{i}(s+\bar{t}, \mathbf{p})=\operatorname{span}\left(l_{1}(T, \mathbf{x}), \ldots, l_{j}(s+\bar{t}, \mathbf{p})\right)$ converges to a fixed subspace $L_{j}(\mathbf{p})$ at least at the rate $\exp \left[-\nabla \mu_{j} \bar{t}\right]$ where $\nabla \mu_{j}$ is the spectral gap. ${ }^{31}$

\section{Approach for Time-Scale \& Differential Geometric Analysis of Optimal Control Problems}

Using the state and costate generated from GPM together with the nonlinear dynamical system theory framework of Ref. 28 and 31, an approach is now synthesized to characterize the time-scales and differential geometry along extremal trajectories of a constrained nonlinear optimal control problem. First, the linearized dynamics along an extremal trajectory are given as:

$$
\dot{\mathbf{v}}=\mathcal{H} \mathbf{v}
$$

where the vector $\mathbf{v}$ is given as

$$
\mathbf{v}=\left[\begin{array}{l}
\mathbf{v}_{\mathbf{x}} \\
\mathbf{v}_{\lambda}
\end{array}\right]
$$

\footnotetext{
${ }^{f}$ While it is true the eigenvalues about an equilibrium point and the Floquet exponents on a periodic orbit define the time-scale structure, we are considering the general non-linear case.
} 
and

$$
\mathcal{H}=\left[\begin{array}{rr}
H_{\boldsymbol{\lambda x}} & H_{\boldsymbol{\lambda} \boldsymbol{\lambda}} \\
-H_{\mathrm{xx}} & -H_{\mathrm{x} \boldsymbol{\lambda}}
\end{array}\right]
$$

is the Hamiltonian matrix. Using Eq. (35), the transition matrix is computed over an appropriate averaging time from Eq. 29, where $\mathbf{F}[\phi(t, \mathbf{p})]$ is replaced by $\mathcal{H}$. The finite-time Lyapunov exponents and vectors are then computed from the singular-value decomposition of the transition matrix taken for an appropriate averaging time.

Once the finite-time Lyapunov exponents and vectors have been computed for a chosen point along the extremal trajectory, the next step is to determine the contribution of the tangent vector, $\mathbf{G}(\mathbf{p})$, to the extremal trajectory along the different subspaces spanned by the Lyapunov vectors. It is known that for Hamiltonian systems, for every direction in space along which the flow is expanding, another direction in space exists along which the flow of trajectories is contracting. ${ }^{\mathrm{g}}$ Therefore, at each point along the extremal trajectory, the tangent space will be decomposed into subspaces that account for the stable and unstable behavior along with subspaces that account for the slow and fast behavior. As a result, we are interested here in determining the dominant effect of the tangent vector both with regard to stability (i.e., whether the solution is expanding or contracting) and the time-scales (i.e., whether the behavior is slow or fast) at a particular point in the phase space.

In order to determine whether the dominant behavior is stable or unstable and slow or fast at a given point along an extremal trajectory, the tangent vector can be decomposed into components in the basis of Lyapunov vectors. First, the components of $\mathbf{G}$ in the Lyapunov vector basis are given as

$$
\mathbf{g}=\left[\begin{array}{c}
g_{1} \\
\vdots \\
g_{2 n}
\end{array}\right]=\left[\begin{array}{c}
\mathbf{1}_{i} \cdot \mathbf{G} \\
\vdots \\
\mathbf{1}_{2 n} \cdot \mathbf{G}
\end{array}\right]
$$

The contribution of $\mathbf{G}$ along the first $m$ directions is then given as

$$
\mathbf{P}=\sum_{j=1}^{m} g_{j} \mathbf{1}_{j}
$$

Note, if $m=2 n$, then $\mathbf{P}$ is the tangent vector expressed in the basis of Lyapunov vectors. Next, by analysis of the Lyapunov exponents, we can determine subspaces of interest. To understand better how the Lyapunov exponents can be used to determine relevant subspaces, consider the following hypothetical example where $\bar{t}=20$ and $n=3$ as shown in Fig. 1 . It is observed for this hypothetical example that a significant separation exists between both the slow and fast exponents and the stable and unstable exponents. There is not, however, a significant separation between the slow-stable or slow-unstable exponents. The small spectral gap of 0.1 between the slow-stable and slow-unstable exponents shows that the averaging time $\bar{t}=20$ is not sufficiently long enough to separate the slow-stable and slow-unstable behavior. A measure of the contribution of the Hamiltonian vector field along the $m$ vectors can then be determined by defining the distance metric $^{35}$

$$
D\left(\mathbf{P}_{\mathbf{p}}, \mathbf{P}_{\mathbf{l}}\right)=\left\|\mathbf{P}_{\mathbf{p}}-\mathbf{P}_{\mathbf{l}}\right\|
$$

where $\mathbf{P}_{\mathbf{p}}$ is the normalized phase space and $\mathbf{P}_{\mathbf{l}}$ is a projection of the normalized phase space into an arbitrary subspace defined above as $\mathbf{P}$ (i.e., the the fast-stable subspace, stable subspace, etc).

${ }^{\text {g}}$ The expanding/contracting nature of Hamiltonian systems is a consequence of Liouville's theorem ${ }^{34}$ 
Because we have normalized the phase space, the metric $D$ returns a value between $0 \leq D \leq 1$. The closer the tangent vector is to an arbitrary subspace, the closer Eq. (40) is to zero and the more orthogonal the tangent vector is to an arbitrary subspace, the closer Eq. (40) is to one. Figs. 2 and 3 provide schematics of the decomposition of a vector field in an arbitrary case and in a case where the vector field has a dominant stable component, respectively. In Fig. 2 it is seen that when $\mathbf{P}_{\text {stable }}$ is subtracted from $\mathbf{G}(\mathbf{p}), \mathbf{P}_{\text {stable }^{\perp}}$ is large. Consequently, the value of the metric $D$ is also large and the tangent vector $\mathbf{P}(\mathbf{p})$ does not lie close to the tangent space of the stable manifold. On the other hand, when $\mathbf{P}_{\text {stable }}{ }^{\perp}$ is small (as shown in Fig. 3), the metric $D$ will taken on a small value. As a result, in this second case the tangent vector $\mathbf{G}(\mathbf{p})$ will lie close to the tangent space of the stable manifold. In both cases, the notion of "close" depends upon a user-specified tolerance.

In general, the stable and unstable (or slow and fast) components of the tangent vector need not be orthogonal to each other. Because a singular value decomposition of the transition matrix results in an orthogonal set of vectors, only some of the resulting vectors will be aligned with the corresponding directions of the different behaviors in the dynamical system. An approach for increasing the number of correctly computed directions of either stable and unstable (or fast and slow) directions is to integrate the transition matrix in both forward and backward time. The following simple two-dimensional example ${ }^{31}$ (shown in more detail later), illustrates the need for bi-directional integration. In the neighborhood of an equilibrium point of a nonlinear dynamical system, the Lyapunov exponents and vectors will converge to the eigenvalues and eigenvectors, which, in general, do not form an orthogonal basis. If a singular value decomposition is performed using a forward integration of the transition matrix, one of the predicted finite-time Lyapunov vectors will be orthogonal. Because we know that the eigenvectors are correct, forward integration is insufficient for obtaining the correct second direction. For this simple two-dimensional example, the approach would be to compute the stable Lyapunov vector in forward time (as described in Ref. 31) and compute the unstable Lyapunov vector in backward time.

A method for capturing the fast-stable behavior in both forward and backward time along a portion of the trajectory and determining if this portion of the trajectory is slow is given as follows. The fast-stable components in forward and backward time are accurately computed by performing a singular value decomposition of the transition matrix. Therefore, the collection of fast stable vectors in forward time,

$$
\mathbf{L}_{f s}=\left(\mathbf{l}_{1}, \mathbf{l}_{2}, \ldots \mathbf{l}_{m}\right)
$$

and fast stable vectors in backward time,

$$
\mathbf{L}_{f s}^{-}=\left(\mathbf{l}_{1}^{-}, \mathbf{l}_{2}^{-}, \ldots \mathbf{l}_{m}^{-}\right)
$$

define the fast stable and fast unstable directions, respectively, along the trajectory. The slow subspace is defined as the intersection the subspaces up to and including the slow parts in forward and backwards time, i.e.,

$$
\mathbf{L}_{f s+\text { slow }} \cap \mathbf{L}_{f s+\text { slow }}^{-}
$$

where $\mathbf{L}_{f s+\text { slow }}$ is the space spanned by the fast stable and slow directions. Then, instead of calculating how close $\mathbf{G}(\mathbf{p})$ is to the tangent space to the slow manifold, the slow manifold is computed directly. In particular, the slow manifold, denoted $S$, can be found by computing where the tangent space to the non-linear dynamical system does not lie in $S^{\perp} . S^{\perp}$ is defined without proof (refer to Ref. 31 for proof) as

$$
S^{\perp}=\mathbf{L}_{f u} \oplus \mathbf{L}_{f u}^{-}
$$

where $\mathbf{L}_{f u}$ represents the space spanned by the fast unstable Lyapunov vectors $\left(\mathbf{l}_{j}, \mathbf{l}_{j+1}, \ldots, \mathbf{l}_{2 n}\right)$. Therefore, the slow manifold is reconstructed ${ }^{36}$ at points along the trajectory by solving the equa-

10 of 26

American Institute of Aeronautics and Astronautics 
tions

$$
\begin{aligned}
& \sum_{i=j}^{2 n}<\mathbf{l}_{i}, \mathbf{G}(\mathbf{p})>=\mathbf{0} \\
& \sum_{i=j}^{2 n}<\mathbf{l}_{i}^{-}, \mathbf{G}(\mathbf{p})>=\mathbf{0}
\end{aligned}
$$

A similar method for determining the slow manifold is via the minimization of the unconstrained objective function $^{31}$

$$
J=\sum_{i=j}^{2 n}\left[<\mathbf{l}_{i}, \mathbf{G}(\mathbf{p})>^{2}+<\mathbf{l}_{i}^{-}, \mathbf{G}(\mathbf{p})>^{2}\right]
$$

By directly computing the slow manifold, it can then be shown where does the solution operate in a neighborhood of it either by visual inspection or measuring the distance of the trajectory from the slow manifold.

Fig. 4 shows a schematic of the algorithm developed in this research that is used to determine the proximity of the Hamiltonian vector field at a given point along an extremal trajectory to tangent spaces of manifolds of interest. In particular, the algorithm described in Fig. 4 consists of the following key steps: (1) solving the optimal control problem via the Gauss Pseudospectral method; (2) mapping the costates of the optimal control problem via the KKT multipliers of the NLP; (3) linearizing the dynamics along the computed extremal trajectory; (4) determining the transition matrix for an appropriate averaging time (where the averaging time needs to be determined iteratively); and (5) determining the time-scale and differential geometry of the tangent space from the Lyapunov exponents and vectors obtained from the singular value decomposition of the transition matrix.

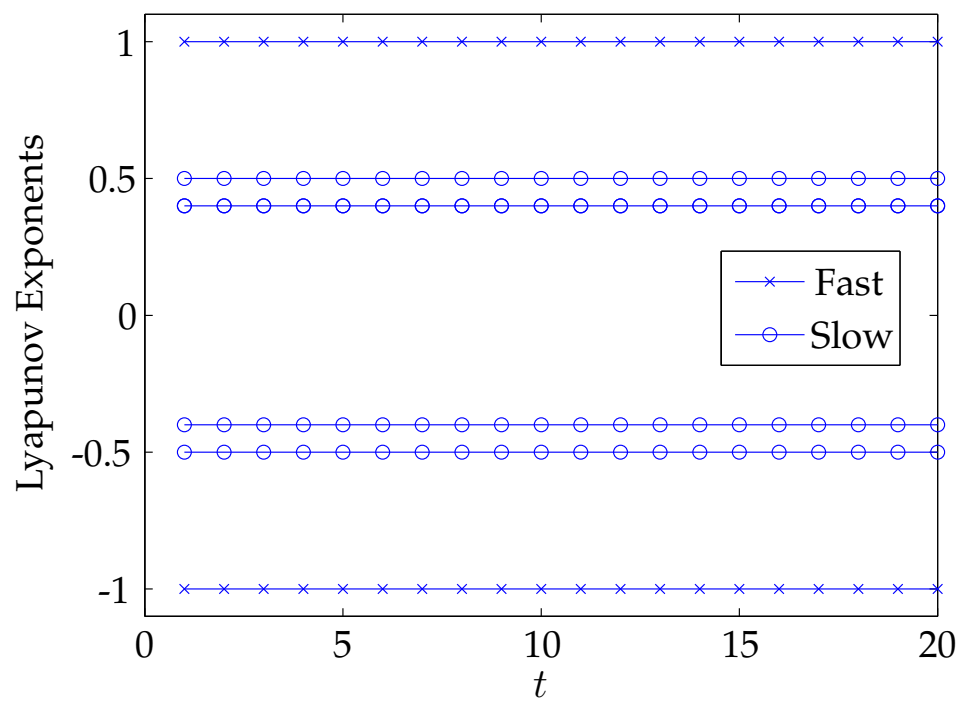

Figure 1. Hypothetical Lyapunov Exponents. 


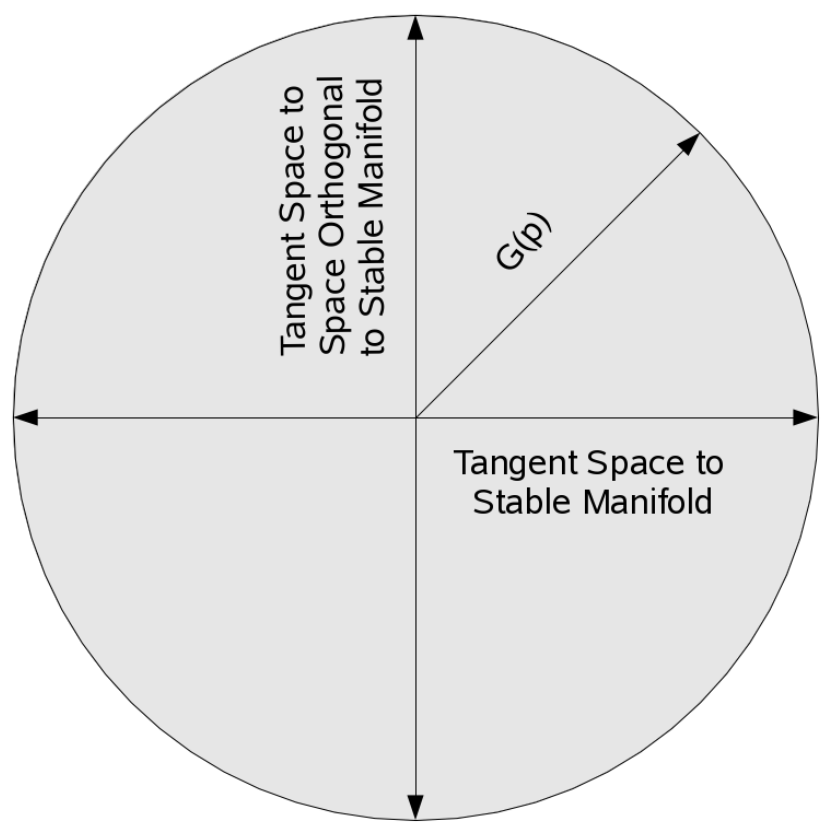

Figure 2. Decomposition of $\mathrm{G}(\mathrm{p})$ into Components That Lie in the TangentSpace of the Stable Manifold and Lie Orthogonal to the Tangent Space of the Stable Manifold.

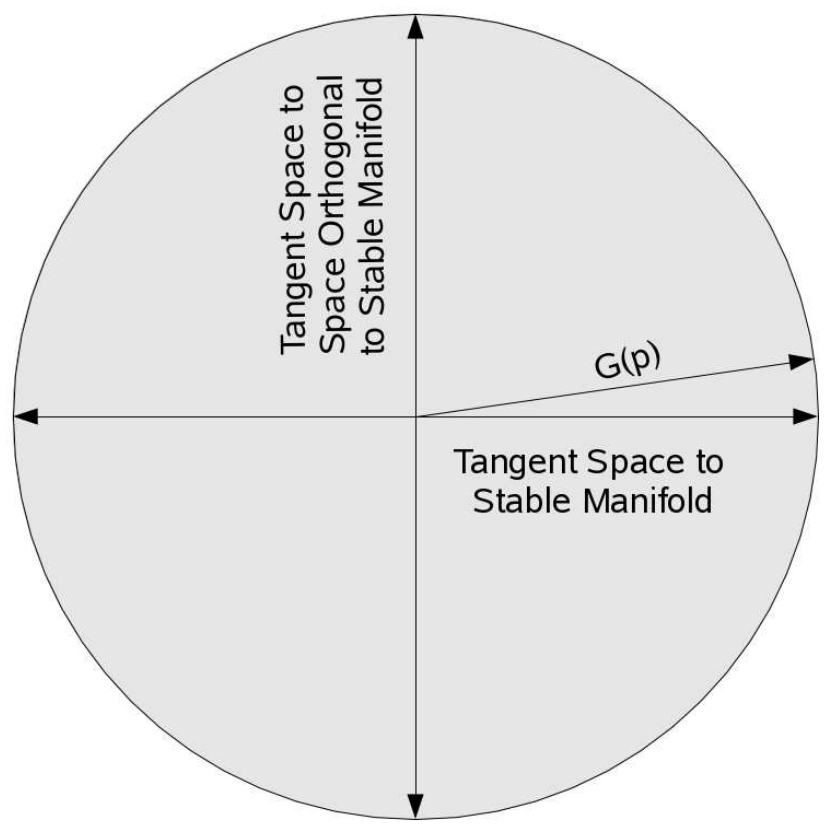

Figure 3. Decomposition of $\mathrm{G}(\mathbf{p})$ into Components That Lie in the TangentSpace of the Stable Manifold and Lie Orthogonal to the Tangent Space of the Stable Manifold for the Case Where the Dominant Behavior is Stable. 


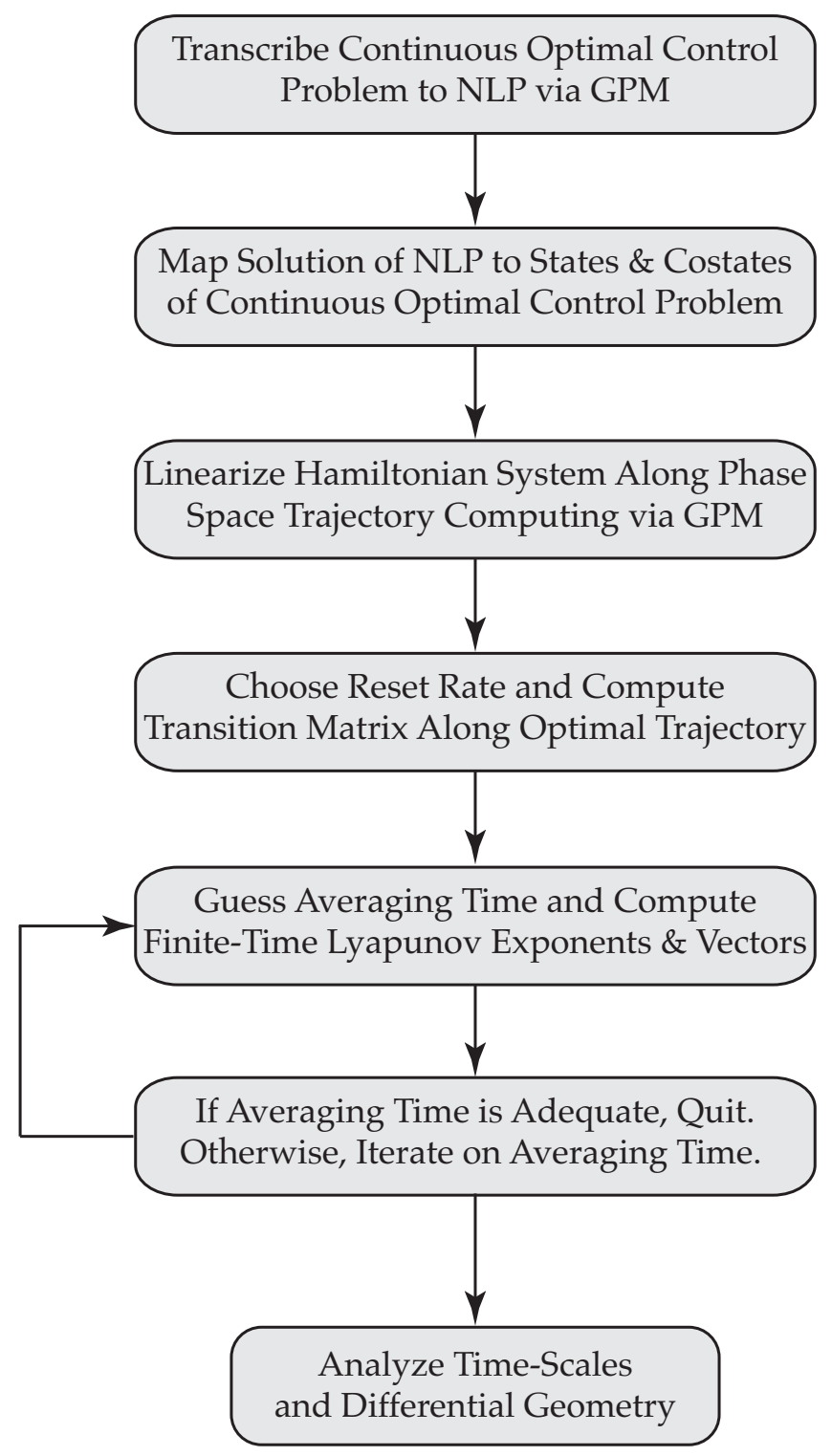

Figure 4. Solution to and Time-Scale Analysis for a General Nonlinear Optimal Control Problem. 


\section{Example 1: Completely Hyper-sensitive Problem}

Consider the following example adapted from Ref. 37. Minimize the quadratic cost functional

$$
J=\int_{0}^{t_{f}}\left(x^{2}+u^{2}\right) d t
$$

subject to the dynamic constraint

$$
\dot{x}=-x^{3}+u
$$

and the boundary conditions

$$
\begin{aligned}
& x(0)=1 \\
& x\left(t_{f}\right)=1.5
\end{aligned}
$$

where the final time $t_{f}$ is fixed. The Hamiltonian is given as

$$
H=x^{2}+u^{2}+\lambda\left(-x^{3}+u\right)
$$

Applying the first-order optimality conditions from the calculus of variations leads to the following Hamiltonian boundary-value problem:

$$
\begin{array}{lll}
\dot{x}=H_{\lambda}=-x^{3}-\lambda / 2 & , & x(0)=1 \\
\dot{\lambda}=-H_{x}=-2 x+3 x^{2} \lambda & , & x\left(t_{f}\right)=1.5
\end{array}
$$

It is known that, for sufficiently large values of $t_{f}$, the optimal control problem of Eqs. (48)-(50) is hyper-sensitive $e^{37}$ because the time interval over which the problem is solved is significantly longer than the minimum rate of contraction and expansion of the Hamiltonian system in the neighborhood of the optimal solution. ${ }^{27,37}$ Moreover, extremal trajectories of the optimal control problem of Eqs. (48)-(50) take on the following three-segment structure as $t_{f}$ increases: (1) a rapidly decaying segment that lies along the stable manifold of the equilibrium point of the Hamiltonian system; (2) a slow segment that lies close to the equilibrium point; and (3) a rapidly growing segment that grows to meet the terminal boundary condition.

In order to demonstrate the hyper-sensitivity, this problem was solved using the Gauss pseudospectral method for 50 nodes using the TOMLAB ${ }^{\circledR 38}$ version of the NLP solver SNOPT. ${ }^{6}$ Because this problem has a two-dimensional Hamiltonian phase space, the hyper-sensitivity can be inferred graphically. First, the evolution of the solution in time is seen in Fig. 5 where the threesegment structure is apparent due to the exponential-like behaviors at the ends with a constant segment in the middle. Furthermore, examining Fig. 7, it is seen that the solution in the phase space looks like two curves connected by a "kink". As it turns out, the curve emanating from the initial condition and ending at the kink is a segment of the stable manifold while the segment starting at the kink and ending at the terminal condition is a segment of the unstable manifold.

Suppose now that one was to assess the structure of the problem, but without any knowledge a priori of the hyper-sensitivity. In this case, suppose we apply the aforementioned framework that uses Lyapunov exponents and vectors to deduce differential geometry. For this problem, computation of the finite time Lyapunov exponents and vectors was conducted with an averaging time of 3 and a reset time on the transition matrix of $0.25^{\mathrm{h}}$. Using the Hamiltonian system of Eq. (52), a comparison is made between the identification of directions corresponding the stable and unstable behavior using the eigenvectors of the Jacobian and using a decomposition based on

\footnotetext{
${ }^{\mathrm{h}}$ To reduce accumulation of numerical errors, the transition matrix is solved for smaller steps, and then these are multiplied together to give the transition matrix over the desired averaging time.
} 


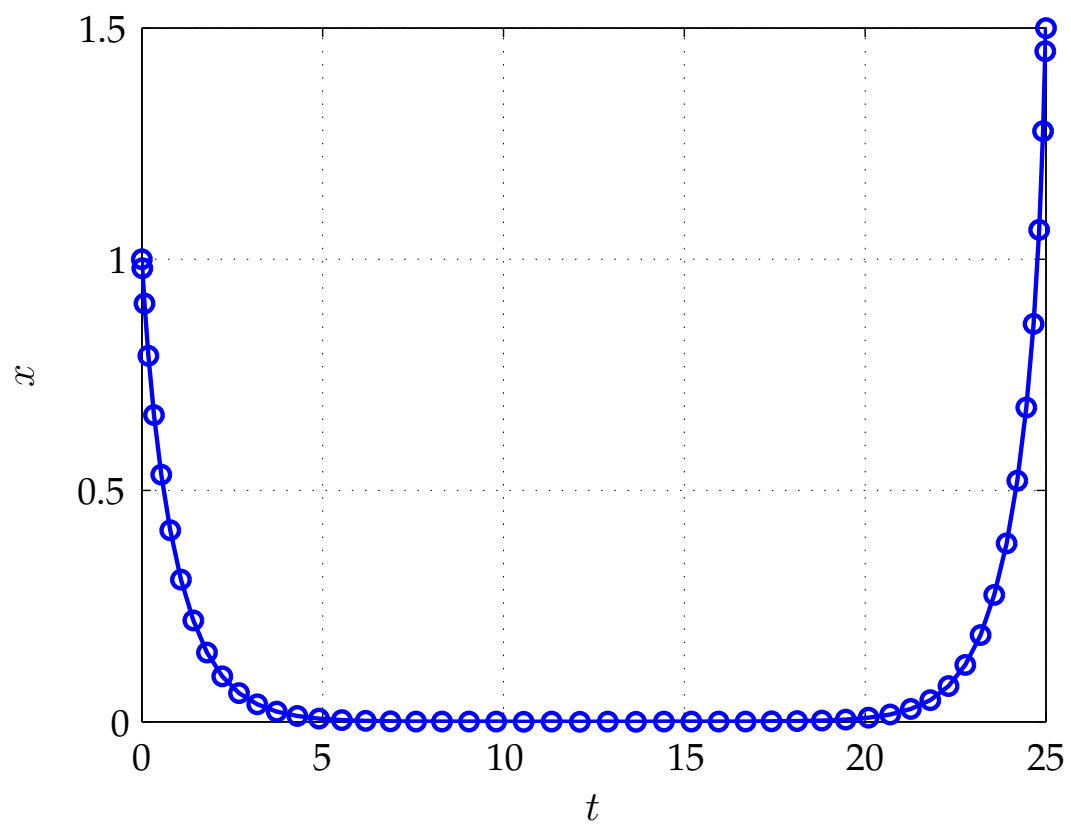

Figure 5. $x(t)$ vs. $t$ for Completely Hyper-Sensitive Problem.

the finite-time Lyapunov vectors. Because the initial part of the trajectory lies extremely close to the stable manifold, it is known that the vector field $\mathbf{G}(\mathbf{p})$ is aligned essentially with the tangent space to the stable manifold (see Ref. 37 for details). The fact that $\mathbf{G}(\mathbf{p}(0))$ lies along the tangent space to the stable manifold is seen in Fig. 8 where $\mathbf{G}(\mathbf{p}(0))$ is tangent to the phase space trajectory. Moreover, because of the known direction of $\mathbf{G}(\mathbf{p}(0))$, any basis that properly identifies the stable direction should have a direction that is tangent to the trajectory at $t=0$. Examining Fig. 8 it is seen that one of the finite-time Lyapunov vectors indeed lies in the same direction as $\mathbf{G}(\mathbf{p}(0))$. Contrariwise, while one of the eigenvectors of $[\partial \mathbf{G} / \partial \mathbf{p}]_{t=0}$ lies in a direction that is somewhat aligned with $\mathbf{G}(\mathbf{p}(0))$, it is seen that this eigenvector is not nearly as good an indicator of the stable behavior as is the stable finite-time Lyapunov vector. Fig. 9 shows that during the initial segment (the green portion which ends at approximately 5), the tangent space to the vector field aligns well with the stable Lyapunov vector. This is expected as the initial segment should operate in close proximity to the stable manifold. At the equilibrium point (the blue portion), the trajectory begins to leave its alignment with the stable Lyapunov vector. Similar results hold for the unstable segment utilizing the backwards time stable Lyapunov vector. Next, Fig. 8 shows a decomposition of the tangent vector at a point $\hat{\mathbf{p}}$ that lies extremely close to the hyperbolic equilibrium point, $\overline{\mathbf{p}}=(0,0)$, of the Hamiltonian system of Eq. (52). As expected, because the equilibrium point is hyperbolic, ${ }^{39}$ the eigenvectors accurately identify both the stable and unstable directions in the neighborhood of $\overline{\mathbf{p}}$. Contrariwise, it is seen that the stable finite-time Lyapunov vector provides reliable information whereas unstable finite-time Lyapunov vector is not reliable. The reason in this second case that the supposed "unstable" Lyapunov vector lies in a direction that is incongruent with the direction of unstable motion is because the singular-value decomposition was computed using a transition matrix that was obtained via a forward time integration of the linearized dynamics. It has been recently discussed (see Ref. 31) that using forward integration alone is generally inadequate in identifying all of the constituent time-scales and stability (i.e., backward integra- 


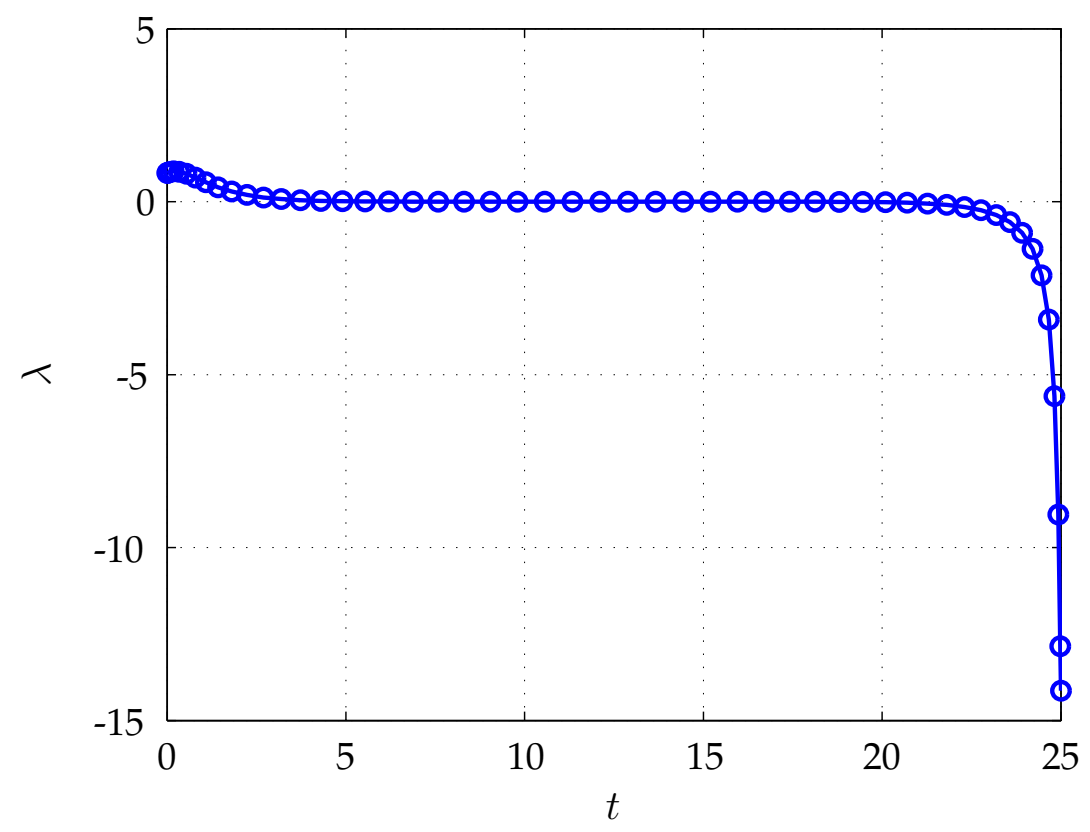

Figure 6. $\lambda(t)$ vs. $t$ for Completely Hyper-Sensitive Problem.

tion is also needed). The issue of forward/backward propagation to identify all of the constituent behaviors is a concept that has come to the attention of the authors only recently (see Ref. 31 for some background on this topic) and is a topic of ongoing research.

Next, using the finite-time Lyapunov exponents, the time-scales can be obtained. Fig. 10 shows the stable and unstable Lyapunov exponents for this problem at various point along the trajectory. It is seen that the Lyapunov exponents converge to approximately \pm 1 , indicating that the timescales are close to unity. Interestingly, the Jacobian of the Hamiltonian system for this problem is given as

$$
\frac{\partial \mathbf{G}}{\partial \mathbf{p}}=\left[\begin{array}{cc}
-3 x^{2} & -1 / 2 \\
-2+6 x \lambda & 3 x^{2}
\end{array}\right]
$$

Evaluating the eigenvalues of the Jacobian at the equilibrium point $\overline{\mathbf{p}}=(0,0)$, we obtain \pm 1 (thus indicating that the time-scales are unity in the neighborhood of the equilibrium point). Because we expect that the time-scales will change gradually as we move away from the equilibrium point, we expect the time-scales to remain somewhat close to unity along the optimal trajectory. Indeed, in this case the finite-time Lyapunov exponents are consistent with the expected result. 


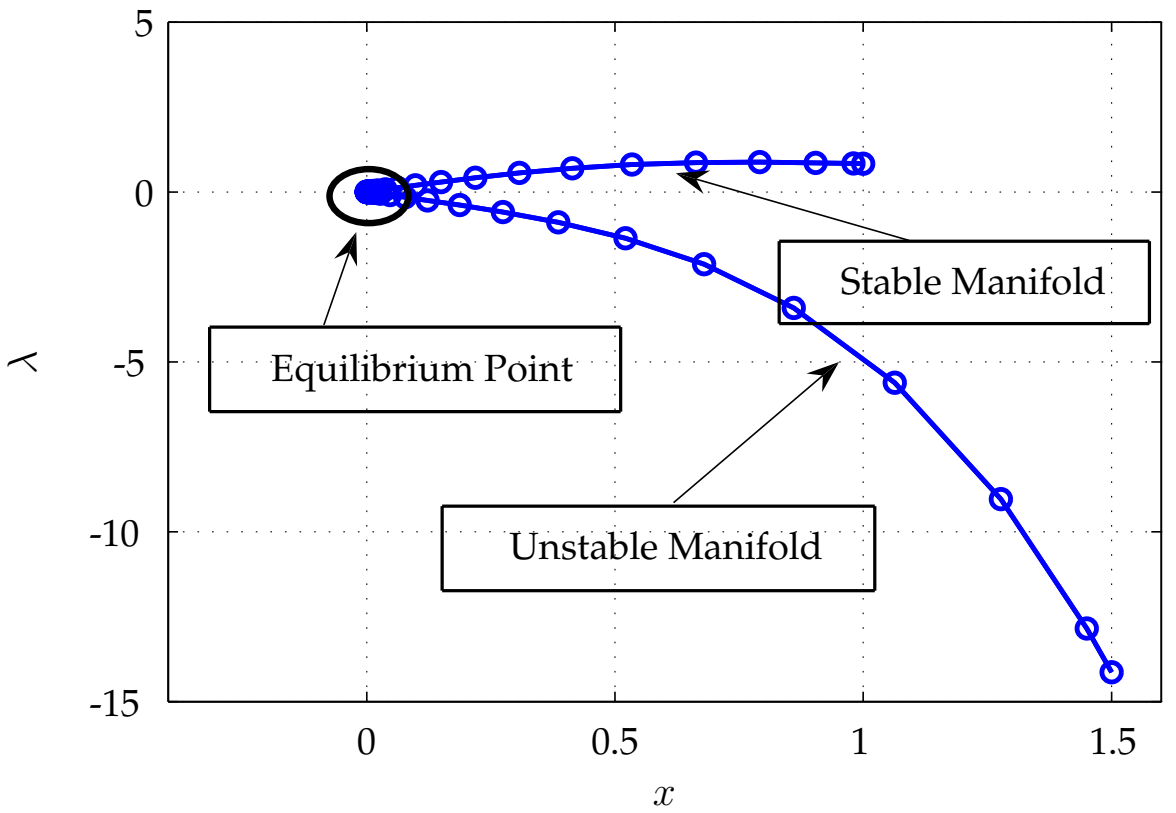

Figure 7. $\lambda$ vs. $x$ for Completely Hyper-Sensitive Problem.

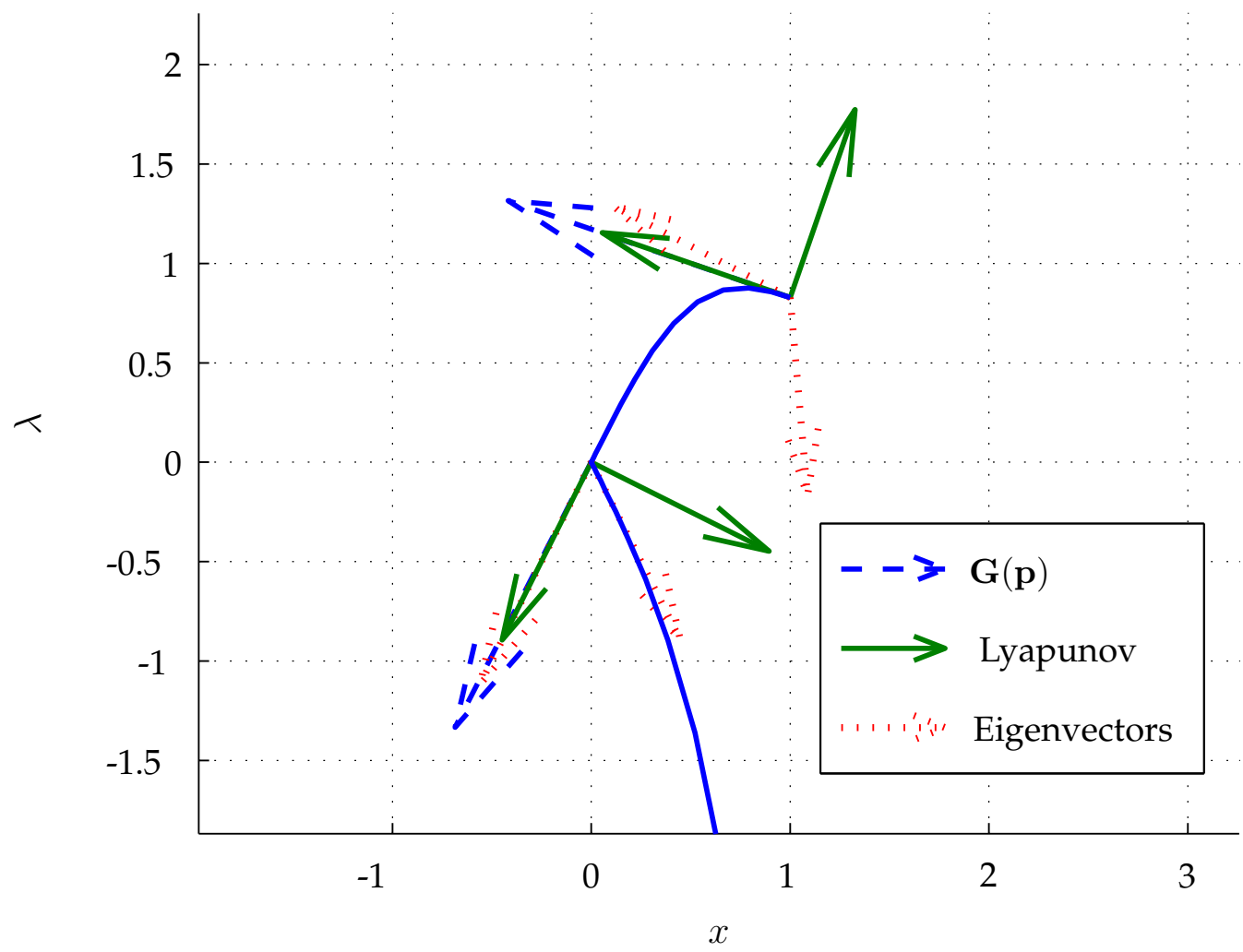

Figure 8. Comparison of Lyapunov Vectors and Eigenvectors (CHSP).

$$
17 \text { of } 26
$$




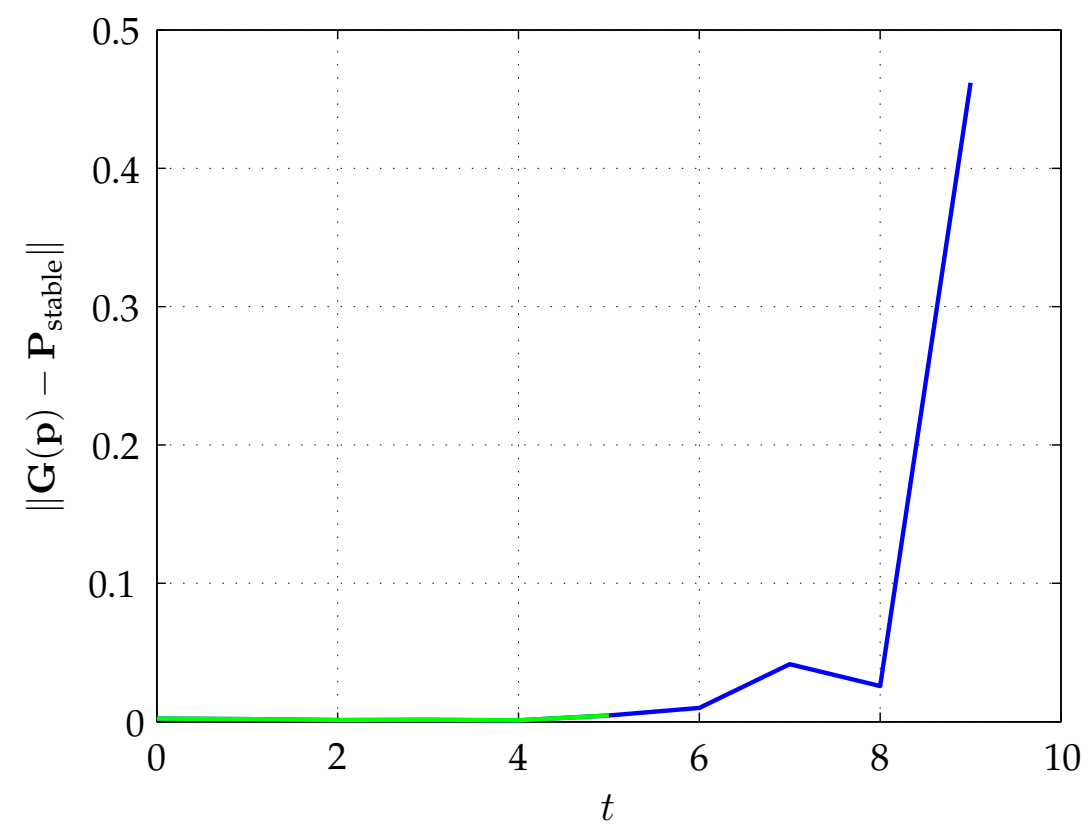

Figure 9. Alignment of the Stable manifold with the Stable Lyapunov Vector for the Completely Hyper-Sensitive Problem.

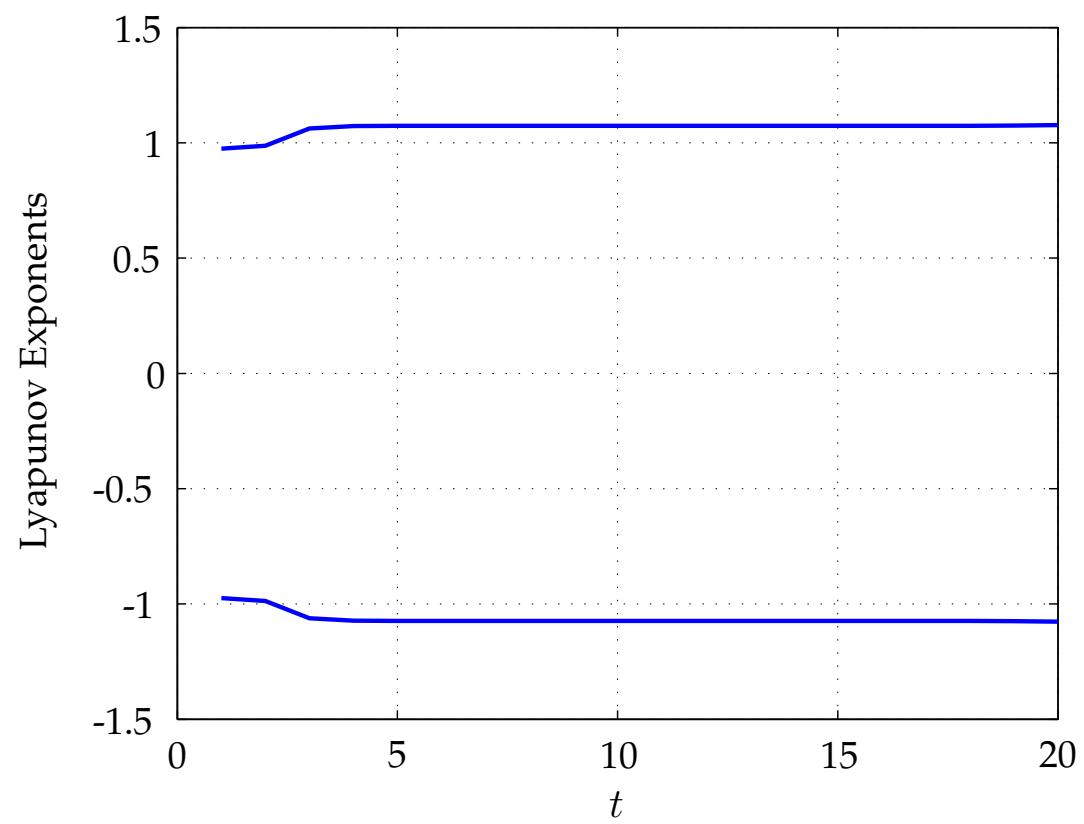

Figure 10. Lyapunov Exponents along the Trajectory for the Completely Hyper-Sensitive Problem.

$$
18 \text { of } 26
$$




\section{Example 2: Chemical Reaction Problem}

Consider the following optimal control problem taken from Ref. 20. Minimize the cost functional

$$
J=\int_{0}^{t_{f}}\left(x^{4}+\frac{1}{2} z^{2}+\frac{1}{2} u^{2}\right) d t
$$

subject to the dynamic constraints

$$
\begin{aligned}
& \dot{x}=x z \\
& \dot{z}=\rho(-z+u)
\end{aligned}
$$

and the boundary conditions

$$
\begin{array}{ll}
x_{0} & =\sqrt{2} / 2 \\
x\left(t_{f}\right) & =1 / 2 \\
z_{0} & =0 \\
z\left(t_{f}\right) & =0 \\
t_{f} & =1
\end{array}
$$

As stated, the optimal control problem of Eqs. (54)-(56) is in the standard singularly perturbed form ${ }^{\mathrm{i}}$ with $x$ and $z$ being the slow and fast variables, respectively. The optimal trajectory for $\rho=10$ is shown in Fig. 11. It is seen that the variable $z$ takes on a three-segment structure with a rapid initial decay from the initial condition, a slow middle segment, and a rapid rise to meet the terminal conditions. What is also seen is that $x$ remains slow throughout the trajectory, which is expected as it is the slow variable. Analyzing the time-scales, Fig. 12 shows the finite-time Lyapunov exponents for $\rho=(10,15,20)$ using an averaging time $\bar{t}=0.5$ and a transition matrix reset time of 0.02. It is seen that the slow Lyapunov exponents (i.e., the red curves marked with diamonds in Fig. 12) do not change significantly as $\rho$ is increased. The slow Lyapunov exponents are actually indistinguishable for each value of $\rho$. On the other hand, the fast Lyapunov exponents (i.e., the blue curves with circles) do change significantly with increasing $\rho$. This behavior in the slow and fast Lyapunov exponents is expected because the dynamics of the variable $x$ remain unchanged as $\rho$ is changed while the dynamics of the variable $z$ increase in speed as $\rho$ increases. While it may seem that a final time of $t_{f}=1$ is small and, thus, should not elicit a time-scale separation in the solution, the fast time-scale in this problem for $\rho \gg 1$ is sufficiently smaller than unity and thus, a time-scale separation appears in the solution. Fig. 13 shows $z(t)$ alongside the slow manifold where the slow manifold is calculated by setting to zero the component of the Hamiltonian vector field in the direction of the fast-unstable Lyapunov vector (where the fastunstable Lyapunov vectors are computed via both forward and backward integration). The slow manifold was calculated by fixing $x$ and $\lambda_{x}$ to their values from the GPM solution and solving for $z$ and $\lambda_{z}$. It is seen that the solution for $z(t)$ lies in the slow manifold in the middle segment, consistent with the fact that a time-scale separation exists and $z$ is the fast variable.

Suppose now that this problem was cast in a form that did not explicitly identify the slow and fast motion (i.e., the problem was not posed in the standard singularly perturbed form). In this case identifying the slow manifold would be less straightforward than was done in the aforementioned analysis. In order to see how one might identify the time-scales for this problem when given the dynamics in a non-standard form, consider the following underdetermined relationship between the variables $x, y$, and $z$ :

$$
x=y^{2}+z
$$

${ }^{\mathrm{i}}$ The standard singularly perturbed form is obtained by setting $\epsilon=1 / \rho$ in the second differential equation. 


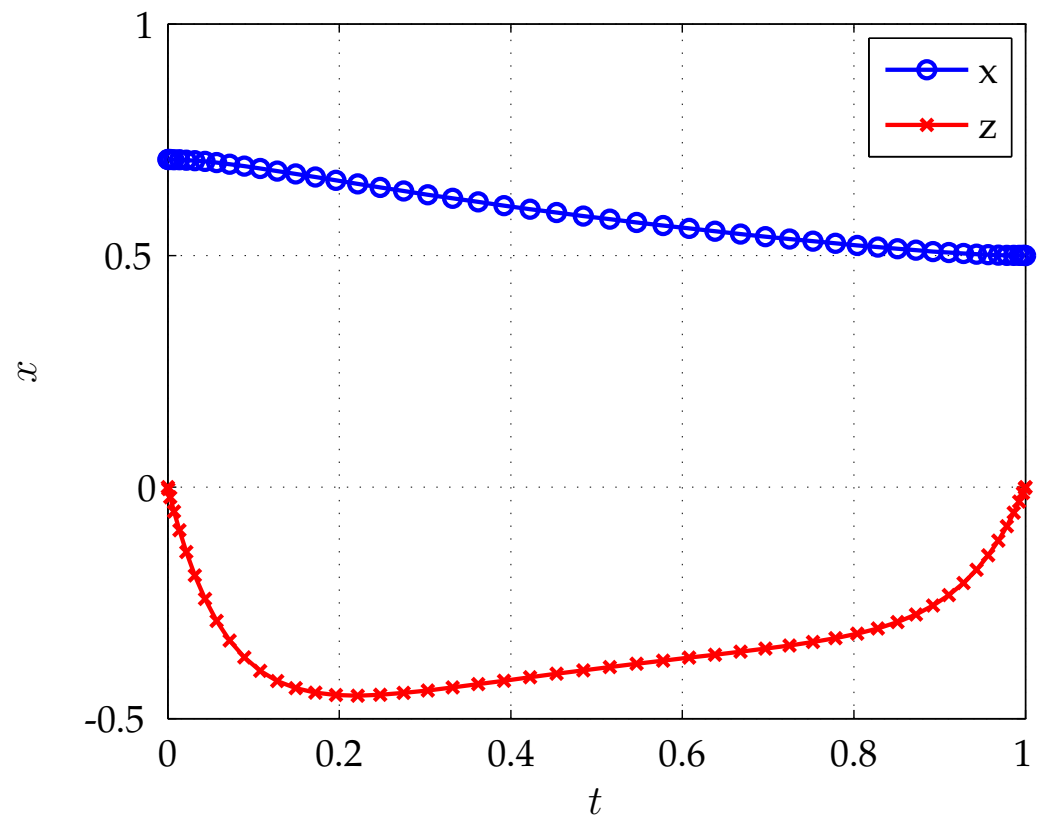

Figure 11. States $x$ and $z$ vs. Time $(\rho=10)$ for Chemical Reaction Example.

Using Eq. (57), the following two new sets of differential equations can be derived:

$$
\begin{aligned}
& \dot{x}=x^{2}-x y \\
& \dot{y}=\frac{x^{2}-x y^{2}}{2 y}-\frac{\rho\left(-x+y^{2}+u\right)}{2 y}
\end{aligned}
$$

and

$$
\begin{aligned}
\dot{y} & =\frac{y^{2} z+z^{2}}{2 y}-\frac{\rho(-z+u)}{2 y} \\
\dot{z} & =\rho(-z+u)
\end{aligned}
$$

The cost functionals corresponding to Eqs. (58) and (59) are given, respectively, as

$$
\begin{aligned}
& J=\int_{0}^{1}\left(x^{4}+\frac{1}{2}\left(x-y^{2}\right)^{2}+\frac{1}{2} u^{2}\right) d t \\
& J=\int_{0}^{1}\left(\left(y^{2}+z\right)^{4}+\frac{1}{2} z^{2}+\frac{1}{2} u^{2}\right) d t
\end{aligned}
$$

It is noted that, for the case of the $(y, z)$ dynamics, no explicit time-scale separation exists between $y$ and $z$.

To analyze how the state space models $(x, y)$ and $(y, z)$ compare to the standard singularly perturbed form $(x, z)$ each problem was solved by the GPM. The optimal trajectories for the $(x, z)$, $(x, y)$ and $(y, z)$ systems is shown in Fig. 11 for 50 LG points with $\rho=10$ (where the relationship of Eq. 57 was used to change between variables when appropriate). The slow manifold was then 


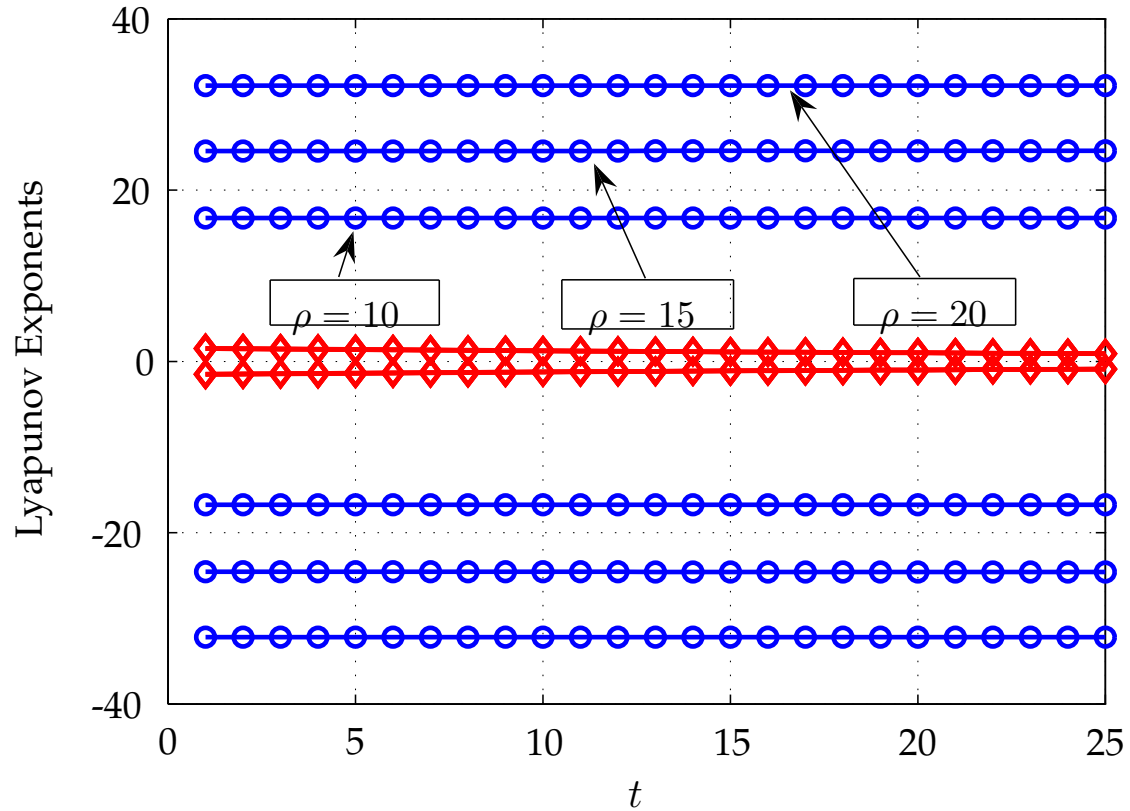

Figure 12. Lyapunov Exponents vs. Time for $\rho=(10,15,20)$ for Chemical Reaction Problem.

calculated with each state variable representation. Then, using Eq. (57), the slow manifold was computed in terms of $z$. Figs. 14 and 15 show the slow manifold for the $(x, y)$ and $(y, z)$ representations, respectively, in terms of the variable $z$. What is seen is that in both the $(x, y)$ and $(y, z)$ cases, the slow manifold is comparable to the one produced from the singularly perturbed form.

It should be noted that all the above slow manifolds were generated using an averaging time of 0.02 to calculate the Lyapunov vectors. As the averaging time increases, the range over which the slow manifold can be calculated shrinks because forward and backwards time are being analyzed simultaneously. For example, for a trajectory of duration 10 time unit and an averaging time of 1 time unit in forward time, the last possible time where the Lyapunov vector can be calculated is at 9 time units because for anytime beyond 9 time units the calculation of a Lyapunov vector will require information beyond the terminal time of the trajectory. In this example, the range over which slow manifold can be analyzed would be from 1 to 9 time units (because in backwards time the last vector is calculated at 1 time unit). No appreciable difference was noticed between the slow manifold calculated with a longer averaging time than one which was calculated with a short one, so the shortest averaging time was used. 

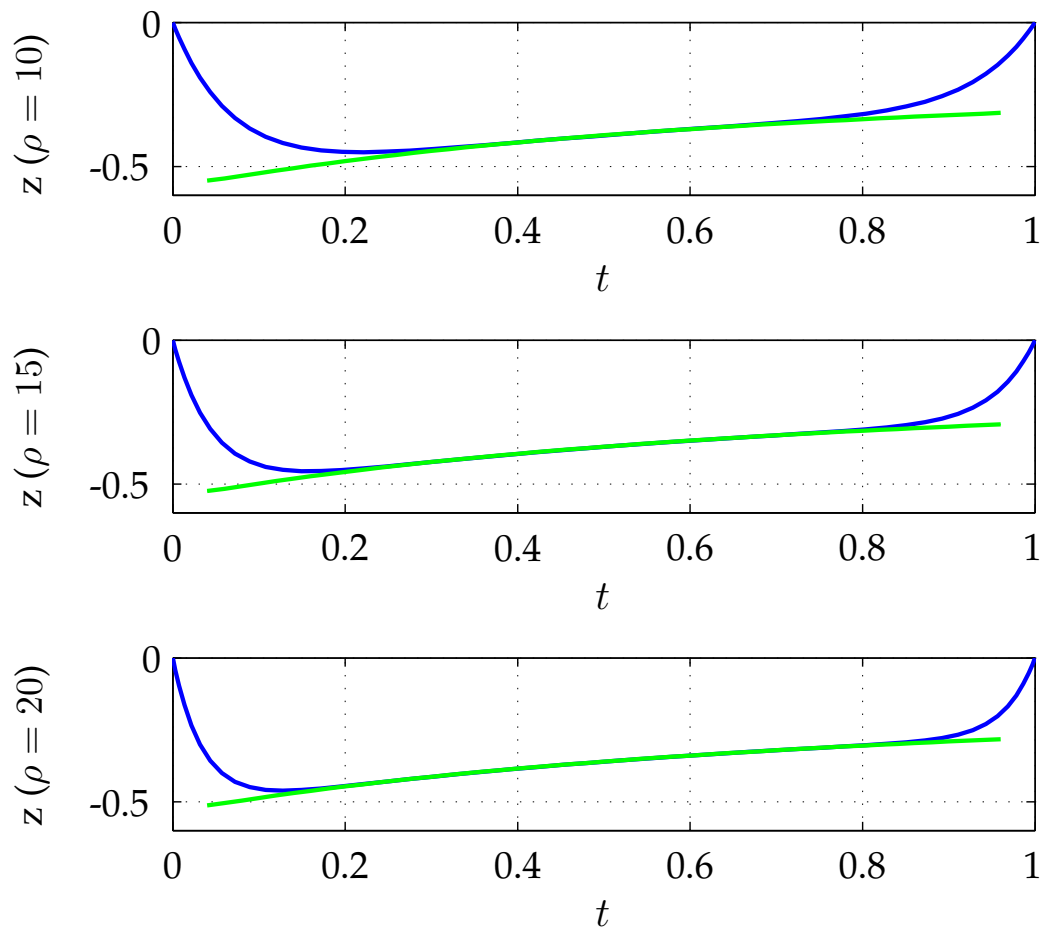

Figure 13. Slow Manifold for the Chemical Reaction Problem for $\rho=(10,15,20)$ Using the Standard Singularly Perturbed Form. 

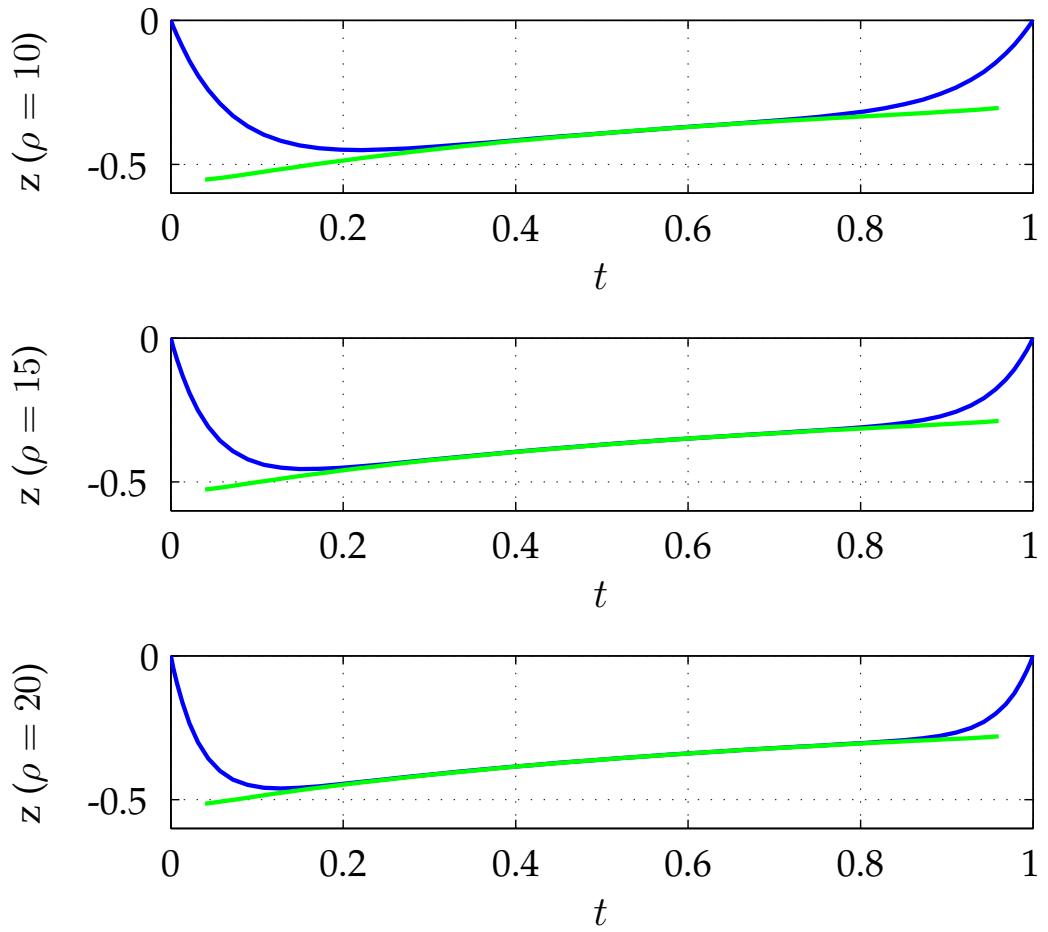

Figure 14. Slow Manifold for the Chemical Reaction Example for $\rho=\mathbf{1 0 , 1 5 , 2 0}$ Using the $(x, y)$ Representation of the Dynamics. 

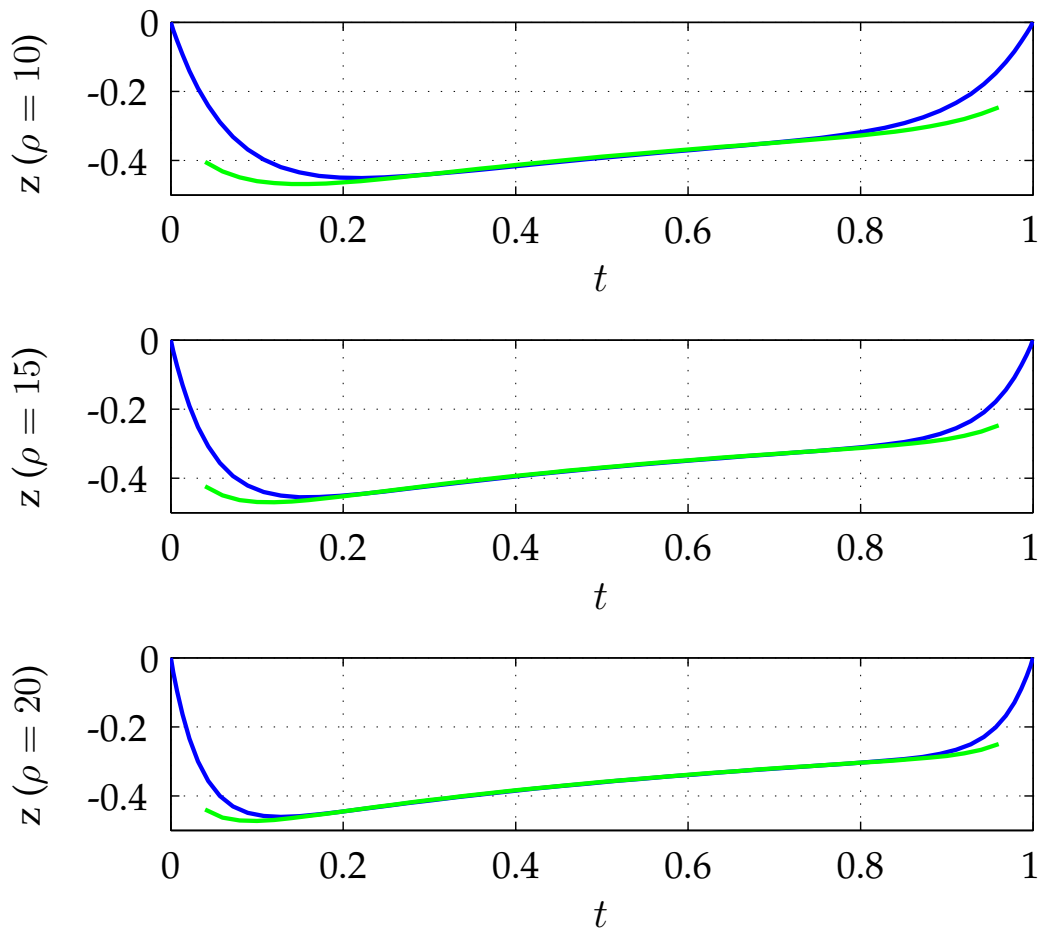

Figure 15. Slow Manifold for the Chemical Reaction Example for $\rho=10,15,20$ Using the $(y, z)$ Representation of the Dynamics. 


\section{Conclusions}

An initial examination has been performed into using pseudospectral methods to infer timescale and differential geometric structure in nonlinear optimal control problems. In particular, the Gauss pseudospectral method has been used to generate accurate Hamiltonian phase space trajectories. These phase space trajectories were then analyzed using a computational differential geometric approach where finite-time Lyapunov exponents and vectors have been computed along the extremal trajectory. The active time-scales and stability has then been determined by examining the dominant direction of the Hamiltonian vector field along the extremal trajectory. The results obtained in this study provide a starting point for time-scale and differential geometric analysis of nonlinear optimal control problems and show the utility of the Gauss pseudospectral method as a viable computational engine to conduct such analysis.

\section{Acknowledgments}

The authors gratefully acknowledge support for this research under funding from The University of Florida.

\section{References}

${ }^{1}$ Betts, J. T., Practical Methods for Optimal Control Using Nonlinear Programming, SIAM, 2001.

${ }^{2}$ Keller, H. B., Numerical Solution of Two Point Boundary Value Problems, SIAM, 1976.

${ }^{3}$ Oberle, H. J. and Grimm, W., "BNDSCO: A Program for the Numerical Solution of Optimal Control Problems," Tech. rep., Institute of Flight Systems Dynamics, German Aerospace Research Establishment DLR, Oberpfaffenhofen, Germany, 1989.

${ }^{4}$ Stoer, J. and Bulirsch, R., Introduction to Numerical Analysis, Springer-Verlag, 2002.

${ }^{5}$ Kirk, D. E., Optimal Control Theory: An Introduction, Dover Publications, 2004.

${ }^{6}$ Gill, P. E., Murray, W., and Saunders, M. A., User's Guide for SNOPT Version 7: Software for Large Scale Nonlinear Programming, February 2006.

${ }^{7}$ Holmstrom, K., Goran, A. O., and Edvall, M. M., User's Guide for Tomlab / Knitro v5.1, April 2007.

${ }^{8}$ Holmstrom, K., Goran, A. O., and Edvall, M. M., User's Guide for Tomlab / SPRNLP / BARNLP, November 2006.

${ }^{9}$ Bazaraa, M. S., Sherali, H. D., and Shetty, C. M., Nonlinear Programming: Theory and Algorithms, WileyInterscience, 3rd ed., 2006.

${ }^{10}$ Elnagar, G., Kazemi, M., and Razzaghi, M., “The Pseudospectral Legendre Method for Discretizing Optimal Control Problems," IEEE Transactions on Automatic Control, Vol. 40, No. 10, 1995, pp. 1793-1796.

${ }^{11}$ Fahroo, F. and Ross, I. M., “Costate Estimation by a Legendre Pseudospectral Method," Journal of Guidance, Control, and Dynamics, Vol. 24, No. 2, 2001, pp. 270-277.

${ }^{12}$ Huntington, G. T. and Rao, A. V., "A Comparison of Accuracy and Computational Efficiency of Three Pseudospectral Methods," Proceedings of Guidance, Navigation and Control Conference, AIAA Paper 2007-6405, Hilton Head, South Carolina, August 2007.

${ }^{13}$ Kameswaran, S. and Biegler, L. T., "Convergence Rates for Direct Transcription of Optimal Control Problems Using Collocation at Radau Points," Computation Optimization and Applications, Vol. 41, No. 1, 2008, pp. 81-126.

${ }^{14}$ Benson, D. A., A Gauss Pseudospectral Transcription for Optimal Control, Ph.D. thesis, MIT, 2004.

${ }^{15}$ Benson, D. A., Huntington, G. T., Thorvaldsen, T. P., and Rao, A. V., “Direct Trajectory Optimization and Costate Estimation via an Orthogonal Collocation Method," Journal of Guidance, Control, and Dynamics, Vol. 29, No. 6, NovemberDecember 2006, pp. 1435-1440.

${ }^{16}$ Huntington, G. T., Advancement and Analysis of a Gauss Pseudospectral Transcription for Optimal Control, Ph.D. thesis, Department of Aeronautics and Astronautics, Massachusetts Institute of Technology, 2007.

${ }^{17}$ Huntington, G. T., Benson, D. A., and Rao, A. V., “Optimal Configuration of Tetrahedral Spacecraft Formations," The Journal of the Astronautical Sciences, Vol. 55, No. 2, April-June 2007, pp. 141-169. 
${ }^{18}$ Huntington, G. T. and Rao, A. V., “Optimal Reconfiguration of Spacecraft Formations Using the Gauss Pseudospectral Method," Journal of Guidance, Control, and Dynamics, Vol. 31, No. 3, May-June 2008, pp. 689-698.

${ }^{19}$ Huntington, G. T., Benson, D. A., How, J. P., Kanizay, N., Darby, C. L., and Rao, A. V., “Computation of Endpoint Controls Using a Gauss Pseudospectral Method," Astrodynamics Specialist Conference, Mackinac Island, Michigan, August 2007.

${ }^{20}$ Kokotovic, P. V., Khalil, H. K., and O'Reilly, J., Singular Perturbation Methods in Control: Analysis and Design, Academic Press Inc., 1986.

${ }^{21}$ Hinch, E. J., Perturbation Methods, Cambridge University Press, 1991.

${ }^{22}$ Lam, S. H., “Using CSP to Understand Complex Chemical Kinetics,” Comb. Science Tech, Vol. 89, 1993, pp. 375404.

${ }^{23}$ Lam, S. H. and Goussis, D. A., “The CSP Method of Simplifying Kinetics,” Int. J. Chem. Kin., Vol. 26, 1994, pp. 461-486.

${ }^{24}$ Lam, S. H., Goussis, D. A., and Konopka, D., “Time-Resolved Simplified Chemical Kinetics Modeling Using Computation Singular Perturbation," AIAA 27th Aerospace Sciences Meeting (AIAA 89-0575), Reno, Nevada, January 1989.

${ }^{25}$ Rao, A. V. and Mease, K. D., "A New Method for Solving Optimal Control Problems," Proceedings of the AIAA Guidance, Navigation and Control Conference, Baltimore, 1995, pp. 818-825.

${ }^{26}$ Rao, A. V., Extension of the Computational Singular Perturbation Method to Optimal Control, Ph.D. thesis, Princeton University, 1996.

${ }^{27}$ Topcu, U. and Mease, K. D., “Using Lyapunov Vectors and Dichotomy to Solve Hyper-Sensitive Optimal Control Problems," Proceedings of the 45th IEEE Conference on Decision and Control, 2006.

${ }^{28}$ Mease, K. D., Bharadwaj, S., and Iravanchy, S., “Timescale Analysis for Nonlinear Dynamical Systems," Journal of Guidance, Control and Dynamics, Vol. 26, No. 2, March-April 2003, pp. 318-330.

${ }^{29}$ Lyapunov, A. M., “The General Problem of Stability of Motion," International Journal of Control, Vol. 55, No. 3, 1992, pp. 531-773.

${ }^{30}$ Lorenz, E. N., "The Local Structure of a Chaotic Attractor in Four-Dimensions," Physica D, Vol. 13D, No. 1-2, 1984, pp. 90-104.

${ }^{31}$ Mease, K. D., Topcu, U., and Aykutlug, E., “Characterizing Two-Timescale Nonlinear Dynamics Using FiniteTime Lyapunov Exponents and Vectors," ArXiv.org, July 2008, pp. 318-330.

${ }^{32}$ Dahlquist, G. and Bjorck, A., Numerical Methods, Dover Publications, 2003.

${ }^{33}$ Trefethen, L. N. and David Bau, I., Numerical Linear Algebra, SIAM, 1997.

${ }^{34}$ Arnol'd, V. I., Dynamical Systems, Vol. 5, Springer, 1991.

${ }^{35}$ Hoffman, K. and Kunze, R., Linear Algebra, Prentice-Hall, Inc., 2nd ed., 1971.

${ }^{36}$ Iravanchy, S., Computational Methods for Time-Scale Analysis of Nonlinear Dynamical Systems, Ph.D. thesis, University of California, Irvine, 2003.

${ }^{37}$ Rao, A. V. and Mease, K. D., “Eigenvector Approximate Dichotomic Basis Method for Solving Hyper-Sensitive Optimal Control Problems," Optimal Control Applications and Methods, 2000.

${ }^{38}$ Holmstrom, K., Goran, A. O., and Edvall, M. M., User's Guide for Tomlab, November 2007.

${ }^{39}$ Guckenheimer, J. and Holmes, P. J., Nonlinear Oscillations, Dynamical Systems, and Bifurcations of Vector Fields, Springer-Verlag, New York, 1990. 